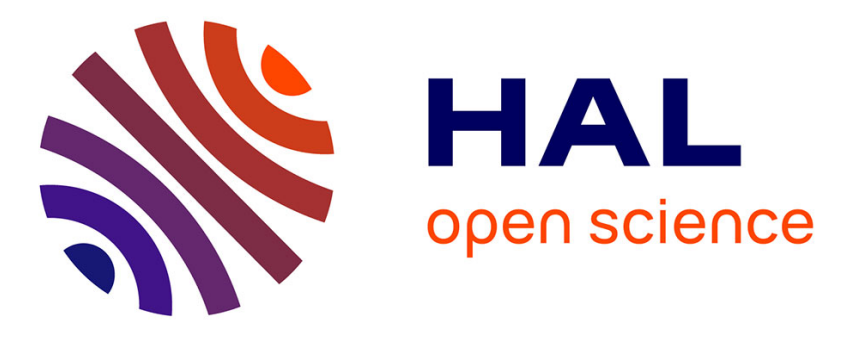

\title{
Application of phase averaging method for measuring kites performance: onshore results
}

Morgan Behrel, Kostia Roncin, Jean-Baptiste Leroux, Frédéric Montel, Romain Hascoët, Alain Nême, Christian Jochum, Yves Parlier

\section{To cite this version:}

Morgan Behrel, Kostia Roncin, Jean-Baptiste Leroux, Frédéric Montel, Romain Hascoët, et al.. Application of phase averaging method for measuring kites performance: onshore results. Journal of Sailing Technology, 2018, pp.2018-05. hal-01889988

\section{HAL Id: hal-01889988}

\section{https://hal-ensta-bretagne.archives-ouvertes.fr/hal-01889988}

Submitted on 8 Oct 2018

HAL is a multi-disciplinary open access archive for the deposit and dissemination of scientific research documents, whether they are published or not. The documents may come from teaching and research institutions in France or abroad, or from public or private research centers.
L'archive ouverte pluridisciplinaire HAL, est destinée au dépôt et à la diffusion de documents scientifiques de niveau recherche, publiés ou non, émanant des établissements d'enseignement et de recherche français ou étrangers, des laboratoires publics ou privés. 


\title{
APPLICATION OF PHASE AVERAGING METHOD FOR MEA- SURING KITE PERFORMANCE: ONSHORE RESULTS
}

\author{
Morgan Behrel \\ ENSTA Bretagne - IRDL UMR CNRS 6027, France \\ Kostia Roncin \\ ENSTA Bretagne - IRDL UMR CNRS 6027, France \\ Jean-Baptiste Leroux \\ ENSTA Bretagne - IRDL UMR CNRS 6027, France \\ Frederic Montel \\ ENSTA Bretagne - IRDL UMR CNRS 6027, France \\ Romain Hascoet \\ ENSTA Bretagne - IRDL UMR CNRS 6027, France \\ Alain Neme \\ ENSTA Bretagne - IRDL UMR CNRS 6027, France \\ Christian Jochum \\ ENSTA Bretagne - IRDL UMR CNRS 6027, France \\ Yves Parlier \\ beyond the sea, France
}

Manuscript received January 26, 2018; accepted September 17, 2018.

\begin{abstract}
This paper describes an experimental set-up aiming to control and measure performances of small leading edge inflatable tube kites, with surface area of less than $35 \mathrm{~m}^{2}$. This set-up can be deployed either onshore or on a dedicated boat. This article focuses on the onshore results. A 3D load cell is used to obtain the position of the kite under a straightline assumption. A wind profiler (SODAR) is deployed to determine the wind speed and direction at the kite position. A specific post-processing of the data is presented, including phase averaging. The guideline of this work is to estimate the variation of the aerodynamic lift coefficient and lift to drag ratio along figure-of-eight trajectories. Results, for a chosen particular case, show a decrease of lift coefficient of about $20 \%$ of the maximum value during turning maneuvers of the kite. The lift to drag ratio evolution along a trajectory is also observed to go through a local minimum during turning maneuvers of the kite in this case. Influence of weight and inertia is highlighted introducing a point mass model of the kite and tethers. Buckling phenomena of the kite inflatable leading edge tube are highlighted and it is shown that they cause control problems.
\end{abstract}

Keywords: kite, phase averaging method, kiteboat, wind profiler.

\section{INTRODUCTION}

With the current continual growth of the global maritime traffic, finding ways to decrease the environmental footprint without higher costs has become a pressing objective. The relevance of a kite as auxiliary propulsion device for merchant ship has been shown by for example Wellicome (1985); Naaijen et al. (2006); Dadd (2013); Erhard and Strauch (2013); Leloup 
et al. (2016). In this context, French sailor Yves Parlier has set-up an innovating project aiming to develop the use of large kites as auxiliary propulsion devices for ships, ranging from small pleasure boats to very large container vessels. The graduate and post graduate school of engineering ENSTA Bretagne - IRDL has been chosen to support the project, and several research actions have been started (seeLeloup et al. (2016), de Solminihac et al. (2018) and Bigi et al. (2018)). One of these aims to provide experimental data to benchmark and validate numerical models developed in the laboratory (see Behrel et al. $(2016,2017)$ ). A few research groups have already undergone trials and measurements on kite, mainly onshore, like Dadd (2013) or Fagiano et al. (2013), and frequently with an electricity production objective (Bormann et al., 2013). Sea trials and measurements have only been conducted by the SkySails team (see Erhard and Strauch (2013)).

With the objective of acquiring experimental data, an experimental setup for measuring kite performances onshore and onboard is developed. Two experimental campaigns are carried out, the first one held onshore in June 2016, and the second held onboard in April 2017 on a dedicated 6-meter-long boat. After a presentation of the experimental set-up, we focus on the exploitation of the onshore results from June 2016. Many figure-of-eight trajectories are recorded during this campaign, and a post processing including phase averaging is developed in order to compute lift coefficient and lift to drag ratio of the kite. One of the objectives is to experimentally benchmark average kite performances predicted by modeling approaches, and to refine the evolution of kite aerodynamic parameters along a figure-of-eight trajectory. Indeed, these variations are not taken into account with the zero-mass model developed by Dadd (2013) or Leloup et al. (2016). However, regarding the deformation of the structure of the kite during a turn it is very probable to get a variation of these aerodynamic parameters, with respect to the turn rate of the kite for example. To the question of the best strategy required to achieve this objective, the answer of an experimental work carried out onshore became rapidly obvious. Indeed, kite performance assessments can be disconnected from boat motions, which is useful to stay focused on the kite modeling itself. Moreover, onshore experiments are easier to achieve, with less consideration about seawater robustness of the experimental set-up, and less human and material resources required.

First, after a short presentation of the reference frames, the paper describes the experimental set-up and its sensors. Secondly, the methodology to do the post-processing is presented. The phase averaging method used here is described. In our case, as the measurement method of the aerodynamic characteristics is not direct, we need to go through a modeling. The classical zero-mass model and the point mass model are used here to assess the importance of inertia effects. Thirdly, the main results are described. The experiments matrix (Tab.1 in section 5) shows the experiments that are effectively carried out. The main entries are the tether length and trim of the kite. Direct outputs are the force measured and its fluctuation during a figure-of-eight fligh as well as the load ratio between the front and the back tethers. Some interesting unexpected results are presented like buckling phenomenon and we will highlight impact on controllability. Finally the evolution of aerodynamic characteristics all along the a figure-of-eight trajectory is shown and, as a first attempt, linear laws with respect to the turning rate are proposed. At the end the results are discussed before concluding.

\section{REFERENCE FRAMES}

The reference frames used in this study are introduced below and detailed with drawings in the following sections. They are in conformity with the ITTC recommendations off the ITTC Quality Group (2014) and directly in accordance with standard ISO 1151-1-4 (1988). They 
are identified with one or two letters, printed in subscript under the normalized vectors $(\underline{x}, \underline{y}, \underline{z})$ forming the axis system. When the considered reference frame needs to be specified, this one is then printed under parenthesis in superscript above the variable: for example, if the vector $\underline{A}_{b}$ is expressed in the reference frame $R_{r e f}$, it will be denoted as $\underline{A}_{b}^{(r e f)}$. In the following Section, "body" stands for the ground station used onshore.

\section{North East Down (NED) Reference Frame}

The earth-fixed reference frame used in this study is the North East Down axis system. It is denoted $R_{n e d}$, and defined with the $x$-axis pointing north, the $y$-axis pointing to the east and the $z$-axis pointing towards the center of the planet.

\section{Heading Reference Frame}

The heading reference frame $R_{\psi}$ is based on the heading angle $\psi$ of the body relatively to the true north direction. It is the result of a rotation about $\underline{z}_{n e d}$ of angle $\psi$ applied to frame $R_{n e d}$, where $\psi$ is the first of the three Euler angles.

\section{Ship Reference Frame}

The ship reference frame is rigidly fixed to the body, and is the result of the two other rotations remaining from the two other Euler angles: the pitch $\theta_{s}$ and the roll $\phi_{s}$. At first, a rotation about $\underline{y}_{\psi}$ of an angle $\theta_{s}$ is applied, followed by a rotation about $\underline{x}_{s}$ of an angle $\phi_{s}$.

\section{Onshore Measurement Wind Reference Frame}

The onshore measurement wind reference frame $R_{w s}$ is the axis system of the wind measurement device when deployed onshore, and not rigidly fixed to the experimental setup. Thus wind measurements are output in this reference frame. This frame is the result of a triple rotation of onshore wind Euler angles $\left(\psi_{w s}, \theta_{w s}, \phi_{w s}\right)$ applied to the heading reference frame $R_{\psi}$. Its origin is located at wind measurement point $W$.

\section{Relative Wind Reference Frame}

For a known relative wind velocity vector $\underline{V}_{W R}$, the relative wind reference frame is defined with the $x_{w r}$-axis co-linear to $\underline{V}_{W R}$ (see Fig.1). Thus the $R_{w r}$ reference frame is the result of a first rotation about $\underline{z}_{\psi}$ of an angle $\chi_{w r}$, followed by a second one about $\underline{y}_{w r}$ of an angle $\zeta_{w r}$. Therefore, $\chi_{w r}$ and $\zeta_{w r}$ angles are the two first Euler angles as defined previously, and transfer matrices $\underline{M}_{\chi_{W R}}$ and $\underline{\underline{M}}_{\zeta_{W R}}$ can also be defined. The relative wind angle $\beta_{W R}$ generally used by sailors, and also used in this study, is related to the angle $\chi_{w r}$ with the following formula:

$$
\beta_{W R}=\chi_{w r}+\pi
$$

\section{Aerodynamic Reference Frame}

The aerodynamic reference frame $R_{a}$ is also defined from the apparent wind on kite, as shown in Fig.2, with the $x_{a}$-axis co-linear to $\underline{V}_{a}$. The $y_{a}$-axis is orthogonal to the plane 


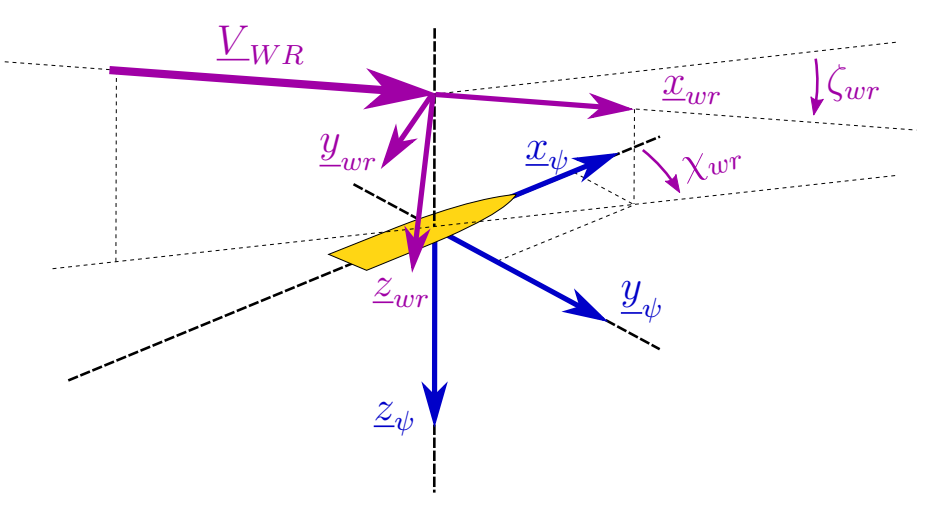

Figure 1: Drawing of the relative wind reference frame $R_{w r}$. The $y_{w r}$-axis and $z_{w r}$-axis are the result of a triple rotation of angles $\left(\chi_{w r}, \zeta_{w r}, 0\right)$, following Euler convention, applied to the heading reference frame $R_{\psi}$.

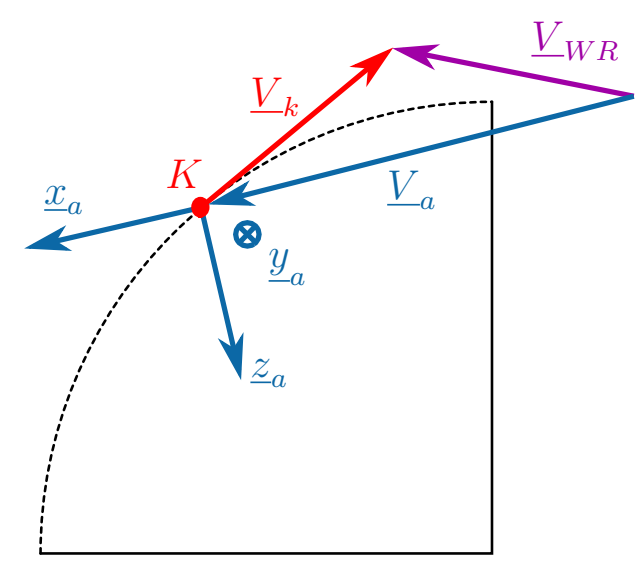

Figure 2: Drawing of the aerodynamic reference frame $R_{a}$. Kite tethers and kite attachment point are not necessarily contained in the plane of the figure.

formed by the vectors $\underline{V}_{a}$ and $\underline{V}_{k}$, and the $z_{a}$-axis is completing the axis system, pointing down. Kite tethers and kite attachment point are not necessarily contained in this plane.

\section{Kite Position}

Kite position is denoted by the point $K$. The position vector $\underline{P}_{k}$ can be expressed in one of the reference frames presented previously. Thus the Fig. 3 is not related to a particular axis system, and presents kite position in a generic reference frame. For the bulk of this study, only kite seen as a point is considered, with no motion of rigid body. This means that only the position of the kite is considered while its attitude is not measured. Therefore, three variables only are requested to position the kite. Because the kite is nearly flying on a sphere, spherical coordinates $(r, \theta, \phi)$ are particularly suitable. However cartesian coordinates $(P x, P y, P z)$ are sometimes necessary.

\section{EXPERIMENTAL SET-UP}

The experimental set-up used for this measurement campaign is based on a kite control box with sensors and actuators, and two additional boxes containing batteries and data acquisition and control system. This trio can be deployed onshore, fixing the kite control box into the ground (Fig.4b), or on board, embedding the system on the boat specifically designed 


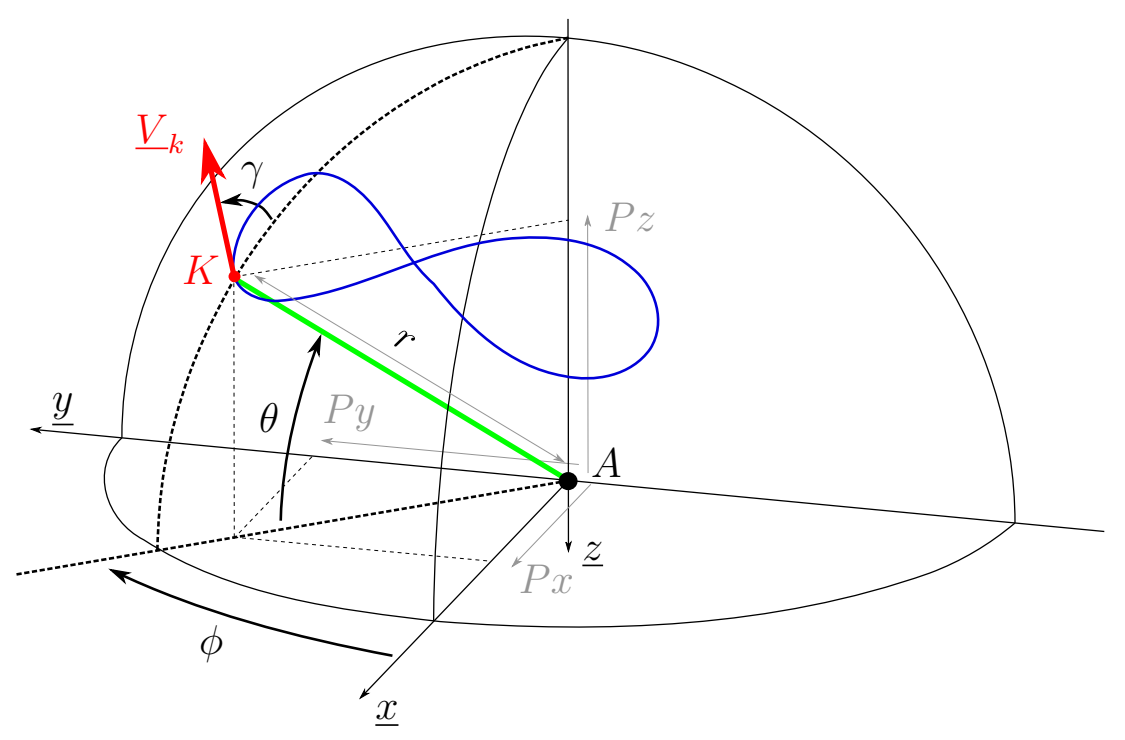

Figure 3: Drawing of the two kite positioning systems: cartesian or spherical. The axis system used for positioning kite can vary depending on application.

for this purpose (Fig.4a). The kite used for this study is a Cabrinha Switchblade $®$, with an area of $5 \mathrm{~m}^{2}$, usually used by kite surfers for leisure sport. Other kites have been tested on the kiteboat. This kite has four tethers, two on each side of the kite: the first two are called front tethers, and have constant length. The two others, called back tethers, have variable length and are used for control purpose. Various lengths of tether were tested during trials, from $25 \mathrm{~m}$ to $80 \mathrm{~m}$.

\subsection{Main sensors}

\subsubsection{Force and Position Measurement}

The major sensor of the experimental device is a three dimensional load cell, providing intensity and direction of the force into the front tethers. This sensor offers the possibility to measure both magnitude and direction of the force with a single sensor and a good accuracy compare to other technics that use angular sensors (Fagiano and Marks (2015); Erhard and Strauch (2013)). These latter techniques need most of the time to add a dimensional load cell in line with the tether, which can affect the sag angle. The load cell choosen in the present study is a TR3D-B-1K built by Michigan Scientific, with a range on each axis of 1,000 pounds $(4,448 \mathrm{~N})$, and a safe overload of $300 \%$ of the full scale. This product is similar to the one used on a previous study Behrel et al. (2016), but with a smaller range of measurement suitable for forces generated by a $5 \mathrm{~m}^{2}$ kite. This sensor has a non-linearity error specified by manufacturer as being under $0.5 \%$ of full scale, and hysteresis and repeatability errors under $0.05 \%$ of full scale each on each of the three components of the load. The error on the load direction is then under $0.3^{\circ}$. The accuracy has been carefully validated in the IRDL laboratory.

For back tethers, due to their variable lengths, another measurement system must be used. This one is based on two simple load cells (Futek LCM200) measuring forces after a return pulley. These load cells have a full scale load of $4,500 \mathrm{~N}$, with a specified non-linearity error under $0.5 \%$ of full scale, an hysteresis error under $0.5 \%$ of full scale and repeatability error under $0.1 \%$ of full scale. Various set-ups for return pulley have been tested, leading to various return angles. These angles were all the time carefully measured to be able to retrieve the real load in tethers. 

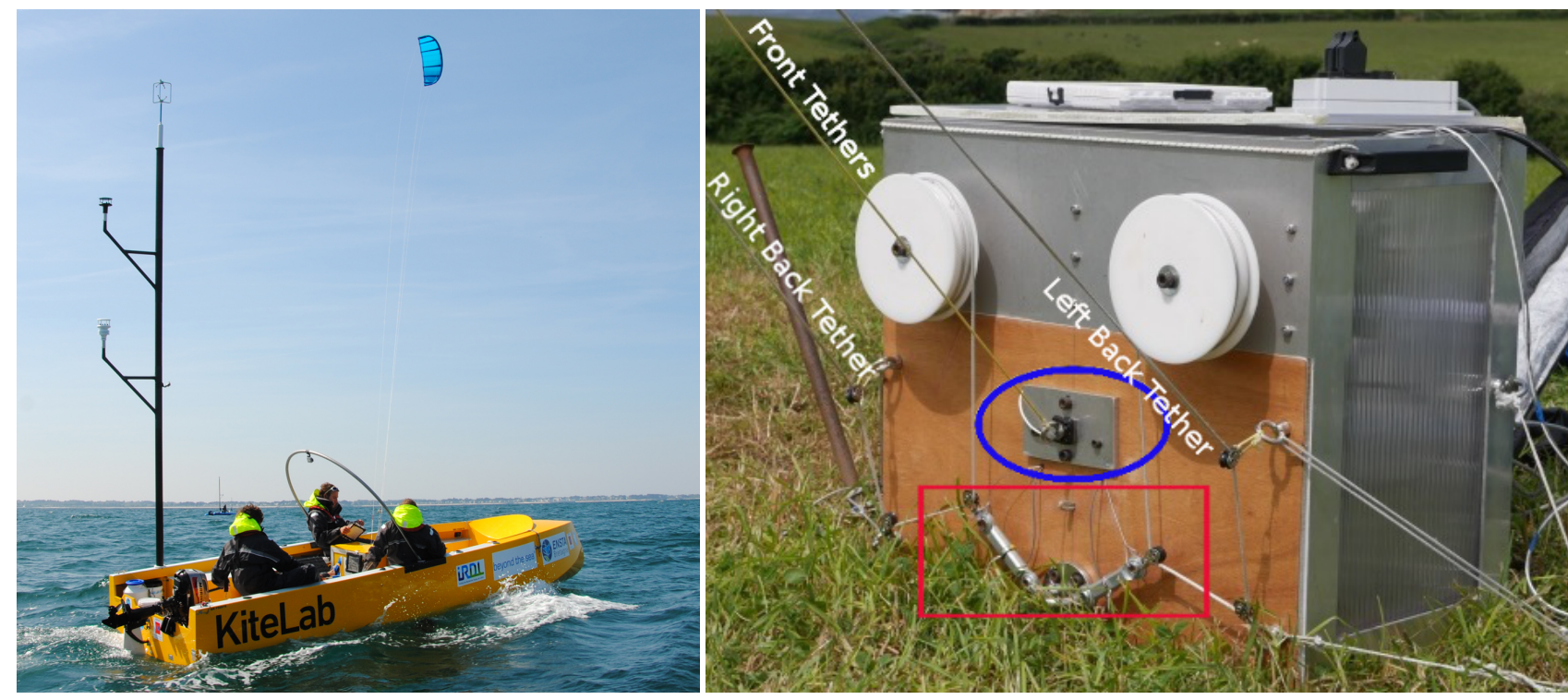

Figure 4: (a) Picture of the Kitelab, the experimental platform specifically designed to carry out measurements on effects and performances of kite propulsion. The $5 \mathrm{~m}$ wind measurement mast is visible at the rear of the boat. The inflatable kite flying over the boat is a 5 $\mathrm{m}^{2}$ one. (b) Kite control and measurement device deployed onshore. The two white drum winches are visible, as well as the 3D front tether load cell (blue ellipse) and the two 1D back tether load cells (red rectangle).

\subsubsection{Onshore Wind Measurements}

Intensity and direction of the wind at kite altitude are important information to get, in order to realize a valuable post processing, as it has been shown in previous work (Behrel et al., 2016). However with a kite flying between $10 \mathrm{~m}$ and $80 \mathrm{~m}$ above the ground, it is difficult to get a wind measurement with a good accuracy at any position of the kite. To deal with this problem, it was decided to use a wind profiler, based on sonic technology (Fig.5). This type of device is called SODAR, for SOnic Detection And Ranging. In our case, the SODAR was able to measure a profile from $13 \mathrm{~m}$ above the ground to $108 \mathrm{~m}$, with one point every $5 \mathrm{~m}$, averaging data over a 5-minute-period. For each point of measurement, the direction, the intensity and the vertical component of the wind velocity were available, but also the standard deviation for each data. It was particularly important to have a wind profiler for these onshore measurements because of the topographic configuration of the field where the trials were carried out. Indeed, it has been observed some variations of the intensity and/or direction and/or vertical intensity of the wind along the altitude that could not have been easy to model.

To catch higher rate wind variation, an ultrasonic three dimensional anemometer METEK USA-1 was also used. This anemometer was put in place on a mast at $8 \mathrm{~m}$ above the ground, and had an acquisition rate of $20 \mathrm{~Hz}$.

In the present study, we choose to use the SODAR as the primary source for all calculations except when it is specifically mentioned that the METEK is used.

\subsection{Kite control system}

\subsubsection{Control And Data Acquisition System}

All the control system and the data acquisition system is driven by a National Instruments compactRIO motherboard, with additional I/O modules, ensuring that all the recorded data 


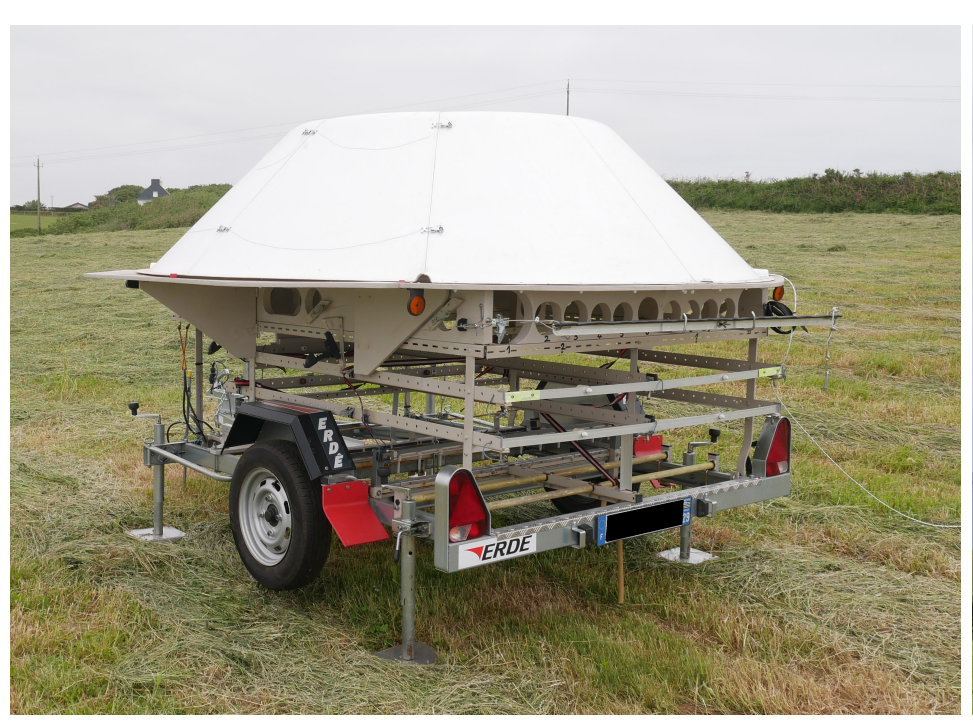

(a)

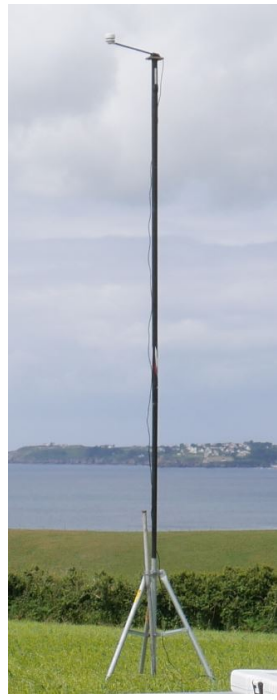

(b)

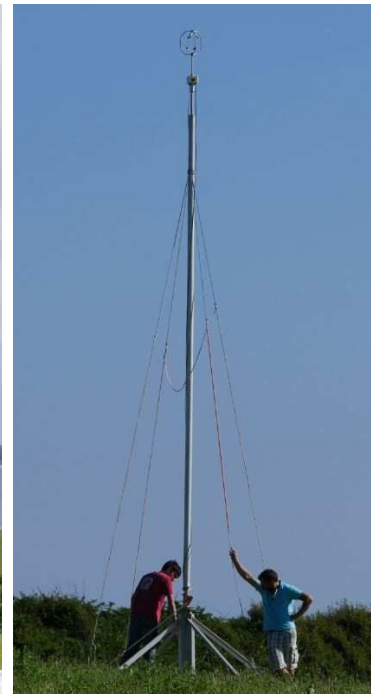

(c)

Figure 5: Onshore wind measurement experimental set-up, based on a SOnic Detection And Ranging wind profiler (a), a 2D ultrasonic anemometer located on a mast, at $4 \mathrm{~m}$ above the ground (b), and a 3D ultrasonic anemometer located on a mast, at $8 \mathrm{~m}$ above the ground (c).

are sampled simultaneously. These modules provide analog and digital inputs, serial ports, and full bridge analog inputs for load cells data acquisition. The whole system is completely programmed using the National Instruments software LabVIEW.

The kite can be steered by applying a difference $\delta$ between the back tether lengths. For this purpose, each back tether is attached to an electric winch, and the winches are controlled in position, thanks to optical encoders with a resolution of 4096 counts per revolution. Thus, for a given differential set-point $\delta$, one winch shall shift by a value $\delta / 2$ and the other winch by a value of $-\delta / 2$. Each winch has a power of 800 watt, and is able to roll in or roll out tethers at a speed of $0.7 \mathrm{~m} \mathrm{~s}^{-1}$. The maximum differential speed is then $1.4 \mathrm{~m} \mathrm{~s}^{-1}$. A power card interfaces the compactRIO and the winches.

\subsubsection{Dynamic Flight Automatic Pilot}

The winches can be controlled by 2 joysticks for a manual control of the kite, but an automatic pilot can be also engaged, enabling the steering of 8-pattern trajectories with good reproducibility. This autopilot is mainly based on Fagiano work (Fagiano et al., 2014). To get a proper functioning of the autopilot, the kite position in the wind window has to be known at any time to ensure a feedback to the controller. More specifically, motion of the kite has to be known, because the kite is undergoing dynamic flight. That means that the kite position data shall be not too noisy to allow the computation of the first order time derivative process leading to the velocity (Eq.16). In our case, the kite position is obtained thanks to the 3D load cell, assuming that the front tethers are straight, and their lengths being carefully measured. According to a verification via a catenary method including aerodynamic drag and weight, this assumption seems reasonable, as it should not cause an error in position of more than $3^{\circ}$ for the reference case presented in the rest of the paper. To reduce noise level on position data, load cell acquisition is done at $10 \mathrm{kHz}$, and then the signal is averaged at a frequency of $200 \mathrm{~Hz}$. After the derivative process, the derivative signal is filtered with a $40 \mathrm{~ms}$ running average filter. 


\subsection{Available data}

Kite data measured during experiments are the force and the direction of the front tether, denoted by $\underline{F}_{m}$, the forces in the left and right back tethers, respectively $F_{b l}$ and $F_{b r}$, and the variable part of the length of the back tethers, which accounts for the position of actuators $\lambda 1$ and $\lambda 2$. The wind data are measured from three different devices, presented in Fig.5. However, the CV7 anemometer used for flight control purpose was only at an altitude of 4 $\mathrm{m}$ and was therefore very disturbed by ground proximity, which could account for difficulties in obtaining symmetrical trajectories. Moreover, a damage of the device has been found at the end of the fieldwork leading to a false wind direction outputted by the sensor. Thus, data from this device are not taken into account in the following work. The two other wind measurements come from the SODAR and the METEK, but the second one was only in place the last two days. Fig. 6 shows some results from the SODAR profiler. These data allow having the wind speed and direction from $13 \mathrm{~m}$ to $108 \mathrm{~m}$ with a $5 \mathrm{~m}$ resolution. Thus, we can interpolate the wind speed and direction at kite altitude. A comparison shows discrepancies about $10 \%$ between the standard ITTC profile and the measurements between $13 \mathrm{~m}$ and $100 \mathrm{~m}$. This exhibits the necessity of such a device for reliable estimation of wind at kite altitude. The results show a significant vertical component of the velocity that directly affect the orientation of the wind window. This must be taken into account for a correct assessment of the aerodynamic characteristics, especially the lift to drag ratio. Some videos and pictures were also recorded using camera such as GoPro, reflex or hybrid camera. Fifteen days of measurement have been carried out, providing $15 \mathrm{~h}$ of relevant flight data.

\section{POST PROCESSING}

The main objectives of the post processing are to obtain estimation of lift to drag ratio and lift coefficient along an 8-pattern trajectory. For this purpose the following equations are computed, and a phase averaging method has been developed, to deal with the low frequency of the SODAR wind measurements.

\subsection{Main equations}

Vectors are expressed into the heading reference frame $R_{\psi}$, as defined in Section 2, unless otherwise noted. All velocities are given with respect to the Earth. At first, forces into front tether are computed in the heading reference frame instead of the frame of the ground station:

$$
\underline{F}_{f}=\underline{\underline{M}}_{\theta_{s}} \underline{\underline{M}}_{\phi_{s}} \underline{F}_{m}^{(s)}
$$

Then, forces into back tethers are added to the front force tether vector, to create the total kite force vector $\underline{F}_{k, A}$ at kite attachment point $A$ on the ground station :

$$
\underline{F}_{k, A}=\frac{\underline{F}_{f}}{\left\|\underline{F}_{f}\right\|}\left(\left\|\underline{F}_{f}\right\|+F_{b l}+F_{b r}\right)
$$

With the hypothesis of perfectly straight tethers with constant lengths $L_{t}$, the total kite force vector at attachment point on the ground station is equal to the opposite of the force $\underline{F}_{k}$ generated by the tethers on the kite at kite position :

$$
\underline{F}_{k}=-\underline{F}_{k, A}
$$

With the same assumption, the kite position $\underline{P}_{k}$ and the kite velocity $\underline{V}_{k}$ can also be computed: 


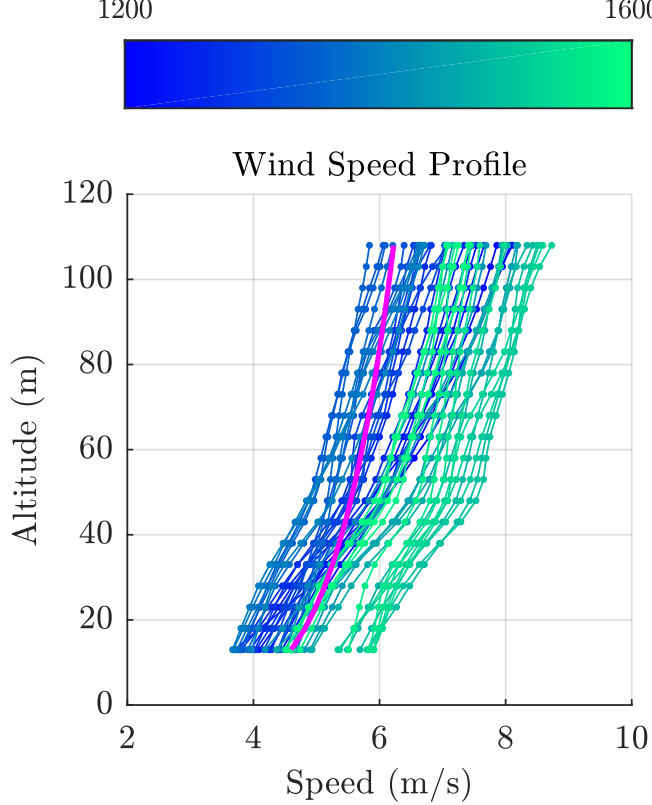

(a)

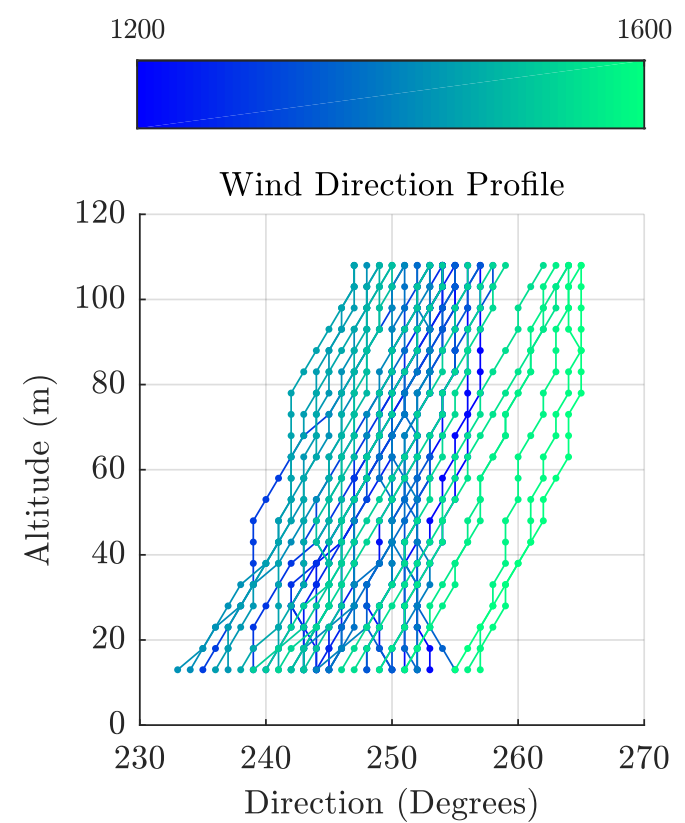

(b)
1200

1600

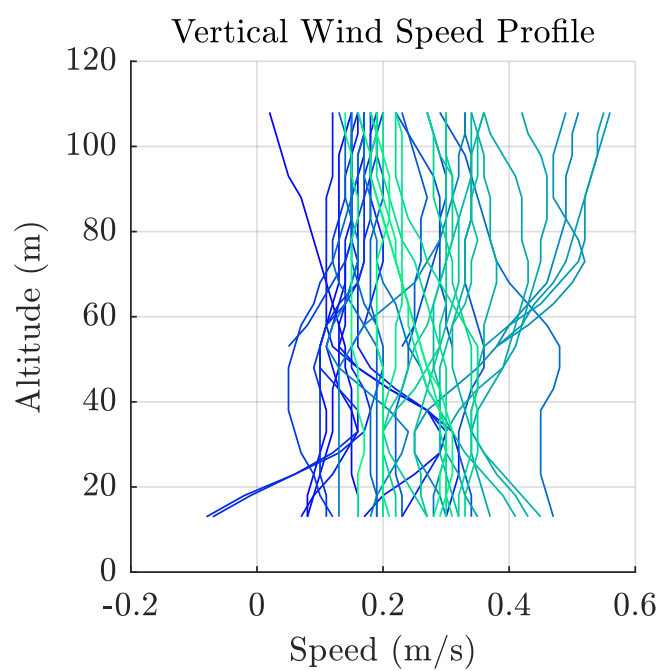

(c)

Figure 6: Example of wind speed profile (a), wind direction profile (b) and vertical wind speed profile (c) measured by the SODAR during the 8th of July between 12:00 and 16:00 UTC (time of each profile depends on color, and is denoted by the colorbar). The magenta line on plot (a) denotes the ITTC profile, calculated from the average wind measured at $13 \mathrm{~m}$ during the period. 


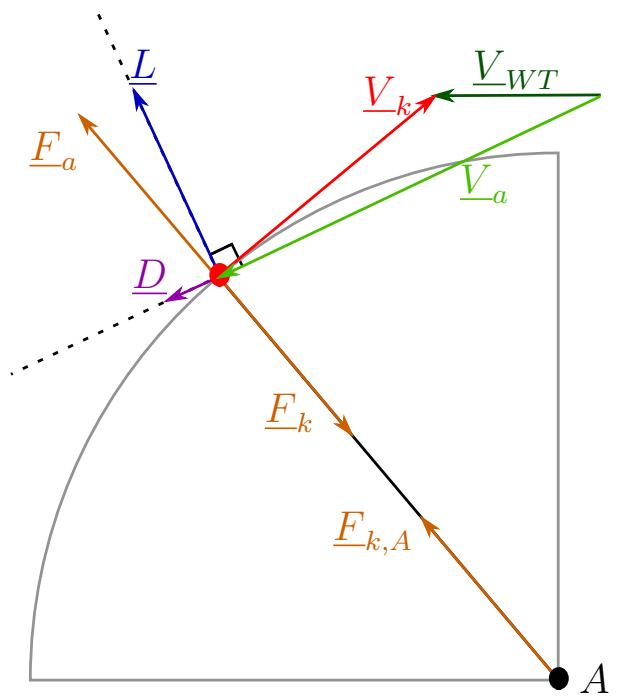

(a) Zero-mass model

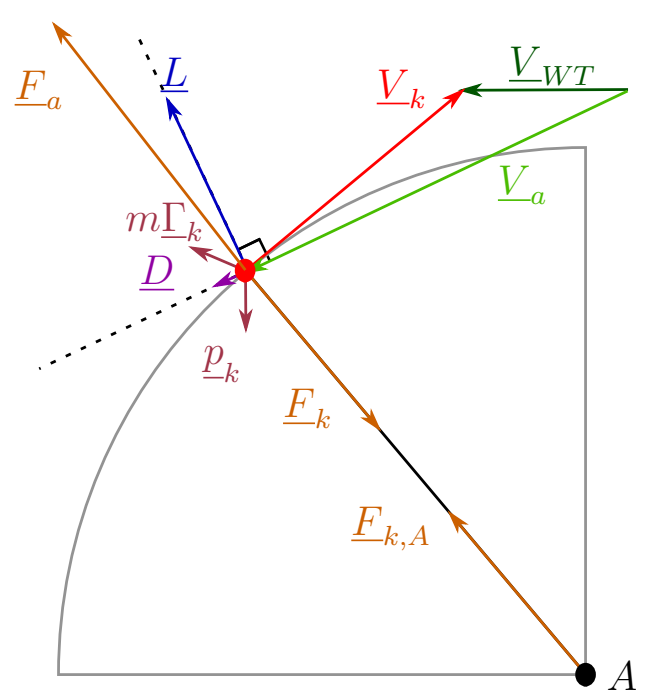

(b) Point-mass model

Figure 7: Force diagrams of the zero-mass model (a) and the point-mass model (b), respectively defined by Eq.7 and Eq.8. For the simplicity of the diagrams, kite force, kite velocity vector, wind velocity vector and kite weight vector are drawn in the same plane; however this is a particular case.

$$
\begin{gathered}
\underline{P}_{k}=\frac{\underline{F}_{f}}{\left\|\underline{F}_{f}\right\|} L_{t} \\
\underline{V}_{k}=\frac{d \underline{P}_{k}}{d t}
\end{gathered}
$$

With the hypothesis of the zero-mass model, the aerodynamic force $\underline{F}_{a}$ generated by the kite is directly equal to the opposite of the force generated by the kite on the tethers at kite position:

$$
\underline{F}_{a}=-\underline{F}_{k}
$$

The force diagram presenting the zero-mass model is given in Fig.7a. However, a second model can be also used, the so-called point-mass model. In this case, the weight of the kite $\underline{p}_{k}$ and inertial forces are taken into account. Impacts on model choice will be discussed in $\bar{S}^{k}$ ection 6 . The force diagram presenting the point-mass model is given in Fig.7b.

$$
\underline{F}_{a}=m \underline{\Gamma}_{k}-\underline{F}_{k}-\underline{p}_{k}
$$

Where $\underline{\Gamma}_{k}$ is the kite acceleration vector, obtained from time derivative of kite speed vector $\underline{V}_{k}$ (Eq.9), and $m$ is the mass of the kite.

$$
\underline{\Gamma}_{k}=\frac{d \underline{V}_{k}}{d t}
$$

In the current case of onshore measurements with a fixed ground station without velocity, the relative wind vector $\underline{V}_{W R}$ is equal to the true wind vector $\underline{V}_{W T}$, and can be get by an axissystem change from the onshore wind measurement reference frame $R_{w s}$ to the heading reference frame $R_{\psi}$. In our case, for all wind measurement devices, $z_{w s}$-axis of $R_{w s}$ reference frame was coincident with the vertical, meaning that $\phi_{w s}$ and $\theta_{w s}$ equals 0 . 


$$
\underline{V}_{W T}=\underline{V}_{W R}=\left(\underline{\underline{M}}_{\psi_{w s}} \underline{\underline{M}}_{\theta_{w s}} \underline{\underline{M}}_{\phi_{w s}}\right) \underline{V}_{W M}^{(w s)}
$$

Knowing the true wind speed and the kite velocity, the apparent wind on the kite $\underline{V}_{a}$ can be computed as follows:

$$
\underline{V}_{a}=\underline{V}_{W T}-\underline{V}_{k}=\underline{V}_{W T}-\frac{d \underline{P}_{k}}{d t}
$$

This apparent wind vector allows to define the aerodynamic reference frame $R_{a}$, as detailed in Section 2. Drag vector $\underline{D}$ can be get by projecting $\underline{F}_{a}$ on $\underline{x}_{a}$ and lift vector $\underline{L}$ is then the difference between $\underline{F}_{a}$ and $\underline{D}$ :

$$
\begin{gathered}
\underline{D}=\left(\underline{F}_{a} \cdot \underline{x}_{a}\right) \underline{x}_{a} \\
\underline{L}=\underline{F}_{a}-\underline{D}
\end{gathered}
$$

The lift to drag ratio $f$ is then obtained by computing the ratio of the norm of the lift and drag vector:

$$
f=\frac{L}{D}=\frac{\|\underline{L}\|}{\|\underline{D}\|}
$$

Finally the lift coefficient $C_{l}$ is also get, with $A_{k}$ the kite area, and $\rho$ the density of the air.

$$
C_{l}=\frac{\|\underline{L}\|}{\frac{1}{2} \rho A_{k} V_{a}^{2}}
$$

\subsection{Time derivative of experimental data}

To compute $\underline{V}_{k}$ and $\underline{\Gamma}_{k}$, time derivative is necessary. A simple centered second-order scheme is used, as following, where $X$ denotes a given signal sampled with a $\Delta t$ time step:

$$
\frac{d X}{d t}(t)=\frac{X(t+\Delta t)-X(t-\Delta t)}{2 \Delta t}
$$

However with experimental data, and particularly using directly the sampling period, this process generates a noise more or less important depending on the quality of the initial signal. Thus a filtering step needs to be added to get relevant results. A zero-phase digital filter built from MATLAB dedicated tools is then applied.

\subsection{Wind estimation at kite position}

As detailed before, the wind at kite altitude can be estimated by two means: the wind measurement done by the METEK sensor, at high frequency at $8 \mathrm{~m}$ above the surface (when available) or the wind profile given by the SODAR, but at low frequency (only 5 min means and standard deviations are available). These two wind sources have been used to compute the post processing, and differences induced in the results are discussed in Section 5 (see Fig.15 a for example). For the METEK case, the wind at measurement point is considered as being equal to the wind at kite altitude. In the case of the SODAR, wind at kite altitude is linearly interpolated in time and space from data provided by the SODAR. 


\subsection{Phase averaging}

The autopilot described in Subsection 3.2.2 is able to perform repeatable 8-pattern trajectories. Nevertheless, due to the variability of the boundary conditions of such full scale outdoor experiments, small variations around a mean periodic trajectory are observed during measurements of several minutes. Therefore, for the analysis of each runs, a conditional phase averaging procedure of the recorded data is applied in order to determine the mean trajectory and all the associated time sampled average characteristics along it (environmental condition, kite control parameters and tensions of the tethers), but also their standard deviations. The present conditional phase averaging process is directly inspired by well-known statistical analysis techniques, which are widely used theoretically or experimentally in the very general field of turbulent flows (Sagaut (2006, Chap.1, Sec. 1.4 ); Wernert and Favier (1999); Yu et al. (2010)).

The chosen detection signal $S_{d}$ is the component of $\underline{P}_{k}$ along the main axis of the cloud of points composed from the whole 8-pattern trajectories of the run. The axis is identified thanks to Principal Component Analysis (PCA): the co-variance matrix $\underline{M}_{\text {cov }}$ is built from the cloud of points (Eq.18 and Eq.19), and then this matrix is diagonalized. The eigenvector associated with the biggest eigenvalue denotes the main axis of the cloud of points, meaning the main axis of the average 8-pattern. This axis is then defined as the $x$-axis of a new reference frame $R_{8}$. The two other eigenvectors complete the reference frame. Each elementary 8-pattern trajectory is then a specific pattern visible on this signal. By centering the signal, each pattern can be isolated by detecting when the signal is crossing zero and becoming negative (Fig. 8 Step 1).

$$
\begin{gathered}
S_{d}=\underline{P}_{k, 1}^{(8)} \\
\forall \vec{X}, \vec{Y} \in \mathbb{R}^{n}, \operatorname{cov}(\vec{X}, \vec{Y})=\frac{1}{n-1} \sum_{p=1}^{n}\left(X_{p}-\mu_{\vec{X}}\right)\left(Y_{p}-\mu_{\vec{Y}}\right) \\
\underline{\underline{M}}_{c o v, i, j}=\operatorname{cov}\left(\vec{P}_{k, i}, \vec{P}_{k, j}\right)
\end{gathered}
$$

From there a set of pieces of the detection signal is available, with as many pieces of signal as the number of patterns initially existing during the run. The average period of the 8-pattern can be computed by averaging the duration of each piece of signal.

Then the detection signal is windowed with the following process: the first point of each window comes from the detection signal and the window length is set equal to the average period of the trajectory. This leads to a set of elementary signals of equal lengths, each associated with one elementary 8-pattern trajectory of the run (Fig.8 Step 2.1). A representative one of these elementary signal is chosen as the reference one (Fig.8 Step 2.2), and the cross correlation of each window with that reference is finally calculated (Fig.8 Step 3.1). This leads to a set of maximum correlation levels and a set of small time shifts for each window to reach this maximum, which is used to slightly correct the beginning of the corresponding windows. This whole process of the detection signal being achieved, all the simultaneously sampled signals recorded in the considered run can be windowed using the obtained final set of the starting time and ending time indices. Finally for a given signal, a mean and a standard deviation can be computed from all the patterns at each sample time of the window (Fig.8 Step 4). Note that the standard deviation on the phase average value $\sigma_{\mu}$ is calculated as follows, under the assumption of independent measurements:

$$
\sigma_{\mu}=\frac{\sigma}{\sqrt{N}}
$$


STEP 1 Average period of the windows, based on a zero crossing search

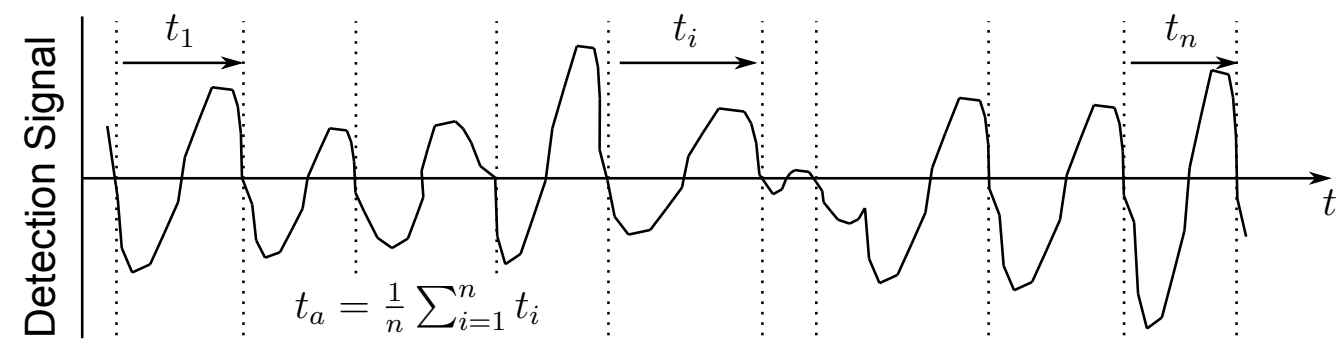

STEP 2.1 Windowing of the signal STEP 2.2 (optional) Reference window selection

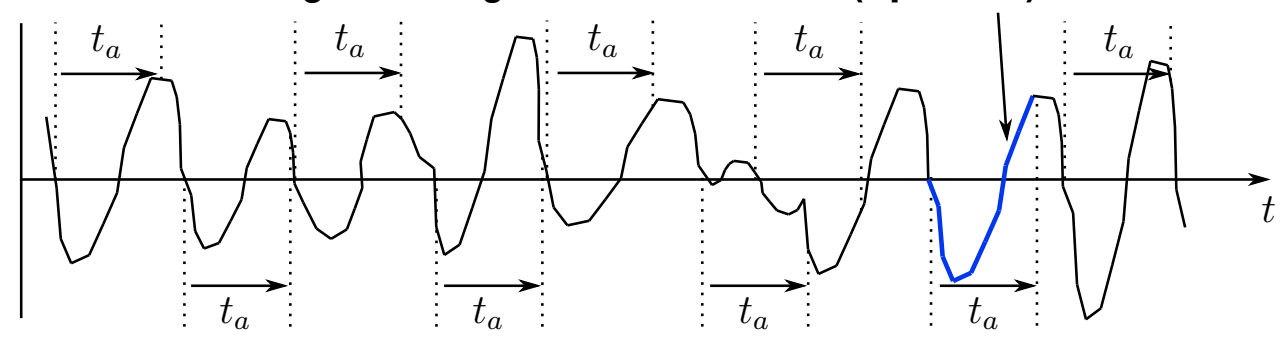

STEP 3.1 (optional) Cross correlation of each window with the chosen reference one
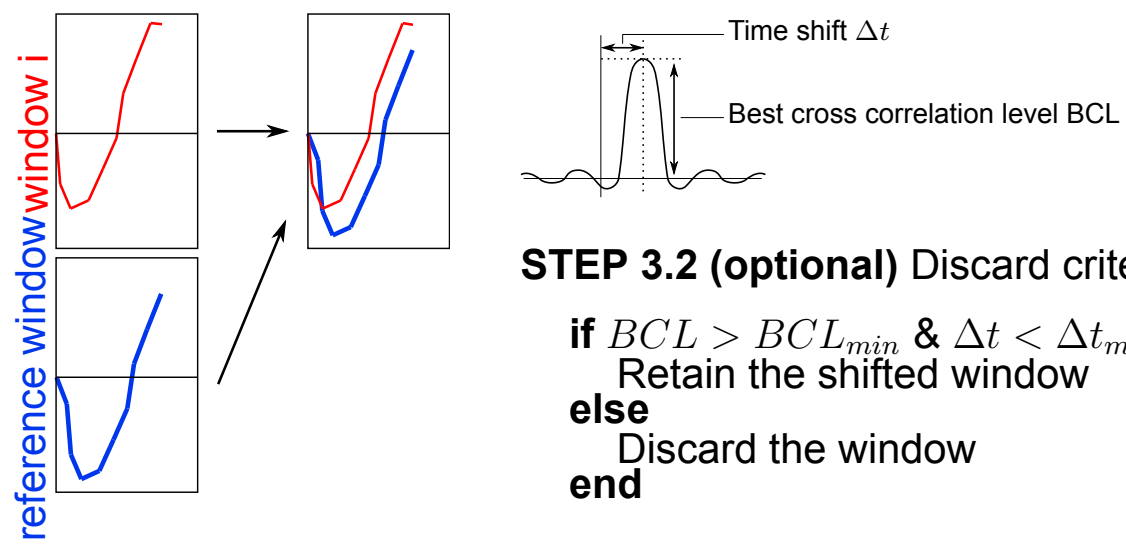

STEP 3.2 (optional) Discard criterion

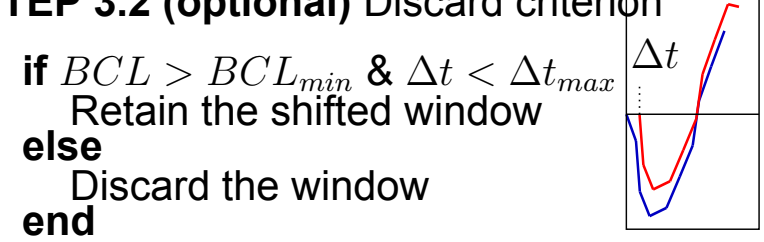

STEP 4 Averaging of the retained shifted windows, for all simultaneously sampled signals

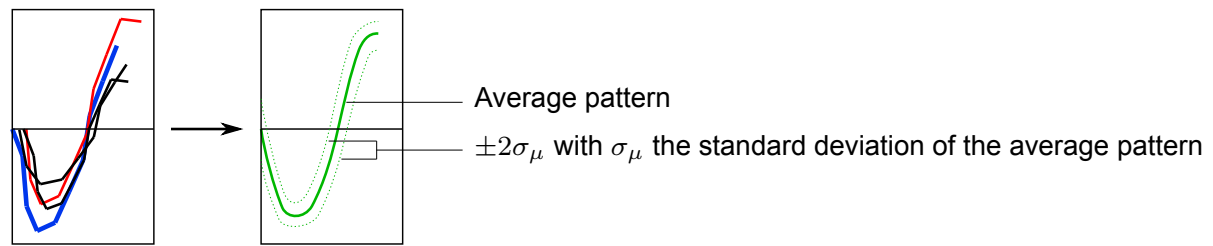

Figure 8: Schematic diagram of the phase averaging method. 
where $\sigma$ is the standard deviation of the considered signal at a given sampled time, and $N$ is the number of patterns used in the phase averaging process for one run.

Moreover, as the cross correlation process returns a level of best correlation for each detected window, it is possible to introduce a conditional criterion to remove spurious sequences from the set (Fig.8 Step 3.2). A limit can be defined in order to only keep windows for which detected pattern have a cross correlation level above a given percentage of the auto correlation level of the reference detection pattern (a level of $70 \%$ is used in the example presented in Fig.9). This cross-correlation check can be done simultaneously on the three components of the front tether force. Thus, not only the geometry of the trajectory is investigated but also the intensity of the force. To ensure a fair comparison, these checks are done on the centered signals normed by the standard deviation of the signals. Moreover, the quality of wind signal is checked, and particularly the SODAR signals. Indeed, some parts of the SODAR signals can be missing, and replaced by NaNs (Not a Number, numeric representation of undefined value). Thus an additional limitation has been introduced to discard all patterns with more than $5 \%$ of $\mathrm{NaN}$ in the SODAR signals.

If the conditional criterion option is selected, the choice of the reference pattern is important and needs to be detailed. In the case of a perfect periodic signal, this choice will have no effect. However the detection signal is rather pseudo periodic with a few extreme events. Therefore a random choice have to be avoided, because the reference pattern could be then an extreme one, and the cross correlation may discard the majority of all other patterns. The option which was finally chosen is to manually select a pattern that seems to be centered in relation to the others. An example of the effect of the phase averaging process is given in Fig.9a, with all the patterns of the runs superimposed on the same plot, for the $y$-axis component of $\underline{F}_{f}$. The result of the method applied on kite position is shown in Fig.9b.

However, in case of a good repeatability, applying such a criteria does not give better results in terms of standard deviation. Furthermore, the application of such a discard criteria is questionable, especially if one wishes to carry out a statistical analysis thereafter. Thus, this criterion was not used in the presented results for the reference case of tab.1.

\section{RESULTS}

The phase averaging procedure has been applied to all data recorded and identified as being potentially relevant, leading to a set a 25 cases, plus some specific cases. Therefore, each run leads to one average 8-pattern trajectory. All these runs were done with the Cabrinha kite, but three different tether lengths were used. Global average data for these runs are given in the Tab.1. These results are analyzed in the following Subsections along several main lines. When a specific plot needs to be shown to illustrate a fact relatively common for all cases, a reference case is then used, which is colored in blue in Tab.1. In the following, an over-right-arrowed quantity denotes a time series, and the associated time line is the one related to the result of the phase averaging method. The letter $\mu$ denotes the average value of a time series.

To compare easily a case to another, the kite force coefficient $C_{k}$ is computed, from the average value of each case:

$$
C_{k}=\frac{\mu_{\overrightarrow{F_{k}}}}{\frac{1}{2} \rho A_{k}\left(\mu_{\overrightarrow{V_{W T}}}\right)^{2}}
$$

The back tether ratio $r_{b}$ represents the part of the force supported by back tethers. The value given in Tab. 1 is the average value $\mu_{\overrightarrow{r_{b}}}$ over a pattern. 


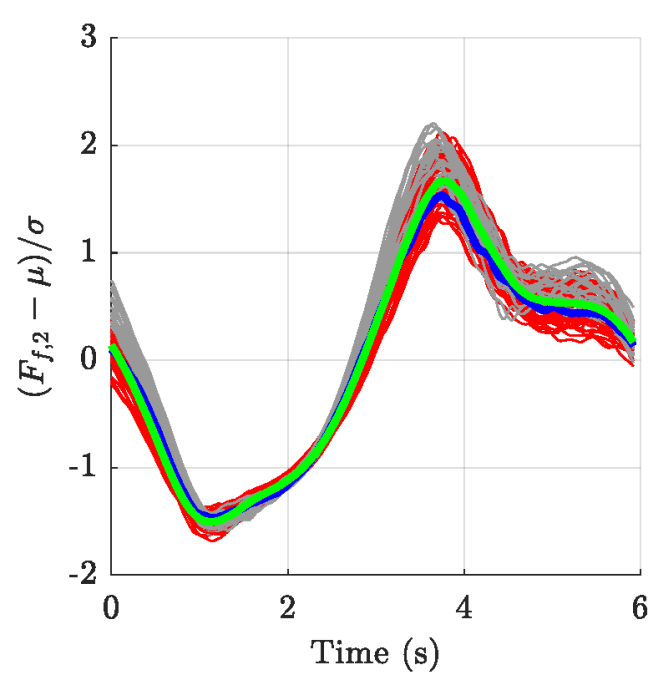

(a)

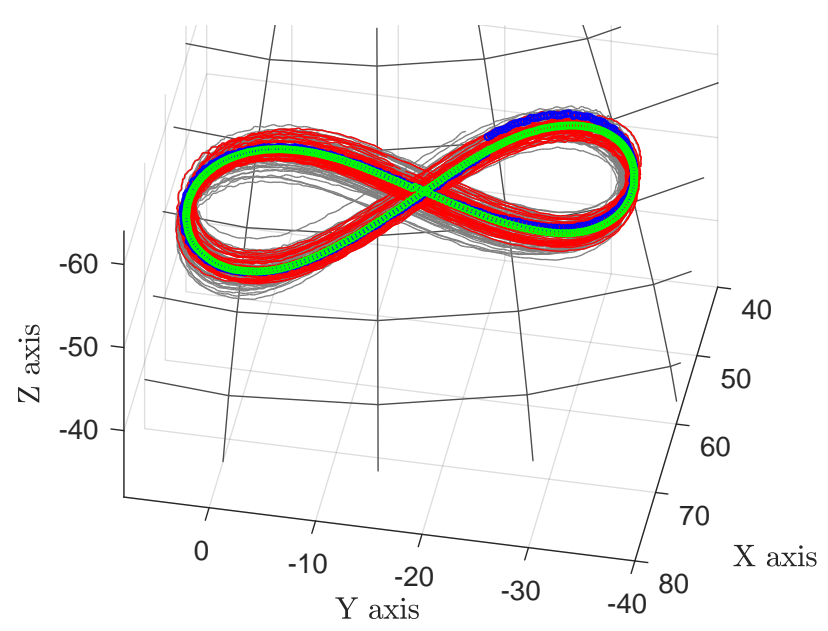

(b)

Figure 9: Plot (a) shows all the patterns of one of the runs in Tab. 1, with colored in blue the reference one, in red the patterns that comply the cross correlation criteria, in gray those which did not, and in green the resulting pattern after the phase averaging. Plot (b) shows all the positions of the kite during the run with the same color code, and the average track resulting from the phase averaging process in green (in the true wind reference frame).

$$
r_{b}=\frac{F_{b l}+F_{b r}}{F_{k}}
$$

The amplitude ratio $r_{a}$ is the amplitude of force along the trajectory.

$$
r_{a}=\frac{\max \left(\overrightarrow{F_{k}}\right)-\min \left(\overrightarrow{F_{k}}\right)}{\mu_{\overrightarrow{F_{k}}}}=\frac{\triangle \overrightarrow{F_{k}}}{\mu_{\overrightarrow{F_{k}}}}
$$

The trajectory width $\triangle t r a j$ is the azimuth amplitude of the trajectory, given in meter, and calculated as follows:

$$
\triangle \operatorname{traj}=L_{t} \triangle \overrightarrow{P_{k, 1}^{(8)}}
$$

In order to select the reference case we used a criterion to choose the run which present the best symmetry. The trajectory is said symmetrical if the phase average of the position of the kite, $\underline{P}_{k}$, is symmetrical related to its "principal plane". The principal plane is defined as the plan containing the center of gravity of the trajectory, $\underline{P}_{k, G}$, and being orthogonal to $\underline{U}_{\sigma, \max }$, the unit vector of the axis according to which the dispersion of $\underline{P}_{k}$ is minimal. Unit vector, which is determined by the Principal Component Analysis (PCA) presented previously in Subsection 4.4. It is then possible to use the following normalized scalar as a symmetrical criterion:

$$
\text { Straj }=\frac{\int_{0}^{P / 2}\left[\left(\underline{P}_{k, c}(t)+\underline{P}_{k, c}(t+P / 2)\right) \cdot \underline{U}_{\sigma, \max }\right]^{2} \mathrm{~d} t}{\int_{0}^{P} \underline{P}_{k, c}(t)^{2} \mathrm{~d} t},
$$

where $P$ is the pseudo-period of the trajectory and $\underline{P}_{k, c}(t)=\underline{P}_{k}-\underline{P}_{k, G}$ is the position vector of the kite related to the center of gravity of the trajectory. For a perfectly symmetrical trajectory, we have $S t r a j=0$ and Straj is growing with the assymmetry of the trajectory. 
Table 1: Summary table of average results for the 25 runs studied. The blue row denotes the reference case (C80_3).

\begin{tabular}{|c|c|c|c|c|c|c|c|c|}
\hline Run Name & $\begin{array}{c}L_{t} \\
(\mathrm{~m})\end{array}$ & $\begin{array}{c}\mu_{\overrightarrow{\mathrm{VWT}_{W}}} \\
(\mathrm{~m} / \mathrm{s})\end{array}$ & $\begin{array}{c}\mu_{\overrightarrow{F_{k}}} \\
(\mathrm{~N})\end{array}$ & $\begin{array}{c}C_{k} \\
(-)\end{array}$ & $\begin{array}{c}\Delta \text { traj } \\
(\mathrm{m})\end{array}$ & $\begin{array}{c}r_{b} \\
(\%)\end{array}$ & $\begin{array}{c}r_{a} \\
(\%)\end{array}$ & Straj \\
\hline \hline C25_1 & 25 & 6.4 & 508 & 4.0 & 38.6 & 19.0 & 99.5 & $3.60 \mathrm{E}-3$ \\
\hline C25_2 & 25 & 6.3 & 448 & 3.6 & 39.0 & 18.7 & 107.7 & $4.30 \mathrm{E}-3$ \\
\hline C25_3 & 25 & 6.1 & 459 & 3.8 & 37.9 & 18.7 & 110.5 & $8.51 \mathrm{E}-4$ \\
\hline C25_4 & 25 & 6.1 & 454 & 3.8 & 35.8 & 19.3 & 102.1 & $2.18 \mathrm{E}-3$ \\
\hline C25_5 & 25 & 6.4 & 454 & 3.5 & 33.2 & 19.5 & 93.1 & $2.92 \mathrm{E}-3$ \\
\hline C25_6t & 25 & 5.6 & 432 & 4.3 & 33.2 & 18.9 & 85.6 & $3.61 \mathrm{E}-3$ \\
\hline C50_1 & 50 & 6.4 & 605 & 4.7 & 59.2 & 21.3 & 83.3 & $1.25 \mathrm{E}-3$ \\
\hline C50_2 & 50 & 5.1 & 795 & 9.8 & 49.5 & 24.1 & 78.3 & $9.00 \mathrm{E}-4$ \\
\hline C50_3 & 50 & 7.0 & 1271 & 8.3 & 72.1 & 27.6 & 104.1 & $9.70 \mathrm{E}-3$ \\
\hline C50_4 & 50 & 7.8 & 1274 & 6.7 & 81.0 & 27.1 & 111.0 & $2.39 \mathrm{E}-3$ \\
\hline C50_5 & 50 & 5.5 & 1169 & 12.3 & 54.3 & 25.8 & 69.4 & $1.57 \mathrm{E}-2$ \\
\hline C50_6 & 50 & 4.6 & 1057 & 15.6 & 62.0 & 26.0 & 76.3 & $1.26 \mathrm{E}-2$ \\
\hline C50_7 & 50 & 4.4 & 1031 & 16.5 & 55.7 & 26.7 & 60.6 & $1.79 \mathrm{E}-2$ \\
\hline C50_8 & 50 & 5.4 & 976 & 10.5 & 60.3 & 26.3 & 76.0 & $1.43 \mathrm{E}-2$ \\
\hline C50_9 & 50 & 5.7 & 659 & 6.5 & 60.6 & 21.9 & 120.1 & $9.94 \mathrm{E}-3$ \\
\hline C50_10 & 50 & 5.3 & 516 & 5.8 & 60.1 & 21.3 & 134.1 & $4.02 \mathrm{E}-2$ \\
\hline C50_11 & 50 & 5.3 & 641 & 7.2 & 62.2 & 22.6 & 100.8 & $7.22 \mathrm{E}-3$ \\
\hline C50_12 & 50 & 5.1 & 669 & 8.1 & 61.5 & 23.0 & 102.8 & $7.47 \mathrm{E}-3$ \\
\hline C50_13 & 50 & 5.0 & 621 & 7.9 & 59.9 & 22.8 & 99.6 & $8.20 \mathrm{E}-3$ \\
\hline C50_14 & 50 & 4.8 & 500 & 6.8 & 59.9 & 22.3 & 119.2 & $8.91 \mathrm{E}-3$ \\
\hline C80_1 & 80 & 5.1 & 942 & 11.4 & 56.1 & 27.5 & 74.2 & $1.25 \mathrm{E}-3$ \\
\hline C80_2 & 80 & 5.3 & 877 & 9.8 & 56.1 & 27.8 & 75.3 & $1.27 \mathrm{E}-4$ \\
\hline C80_3 & 80 & 5.0 & 834 & 10.3 & 55.2 & 27.9 & 80.9 & $4.39 \mathrm{E}-5$ \\
\hline C80_4 & 80 & 5.6 & 1124 & 11.3 & 82.7 & 30.1 & 70.9 & $2.58 \mathrm{E}-3$ \\
\hline C80_5 & 80 & 6.0 & 1077 & 9.4 & 94.4 & 30.2 & 72.9 & $7.11 \mathrm{E}-3$ \\
\hline
\end{tabular}




\subsection{Kite loading}

\subsubsection{General case}

As visible in Tab.1, the average force generated by the kite is between 400 and $1300 \mathrm{~N}$, leading to a wing load under $300 \mathrm{~N} / \mathrm{m}^{2}$, so far under the initially target value of $1 \mathrm{kN} / \mathrm{m}^{2}$ Leloup et al. (2016). However this is the average value on a pattern, and the instantaneous value along the trajectory is far from constant, as displayed in the last column of the table: the variation is about $100 \%$ of the average value of the run. This is also illustrated in Fig.10a. The maximum kite force values occur generally at the end of the quasi-straight part of the trajectory, whereas the minimum values are located at the end of the turn, as shown in the 3D plot presented in Fig.10b. $C_{k}$ can be regarded as an efficiency coefficient. Important variations from 3.5 to 16.5 can be observed in Tab.1 for $C_{k}$. This coefficient depends on the kite trim but also on the position of the kite in the wind window. According to the zero mass model, for a kite with a lift to drag ration of 5 , the $C_{k}$ coefficient would vary by a ratio from 1 to 25 between the edge and the full power point of the wind window. Therefore, it can be observed in Tab.1 that $C_{k}$ gets higher when the lines are longer because the kite gets then closer in average to the full power point.

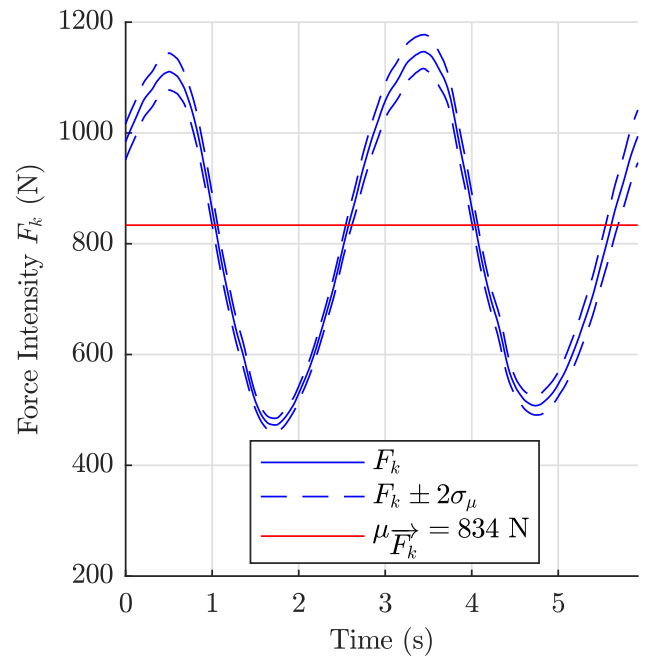

(a)

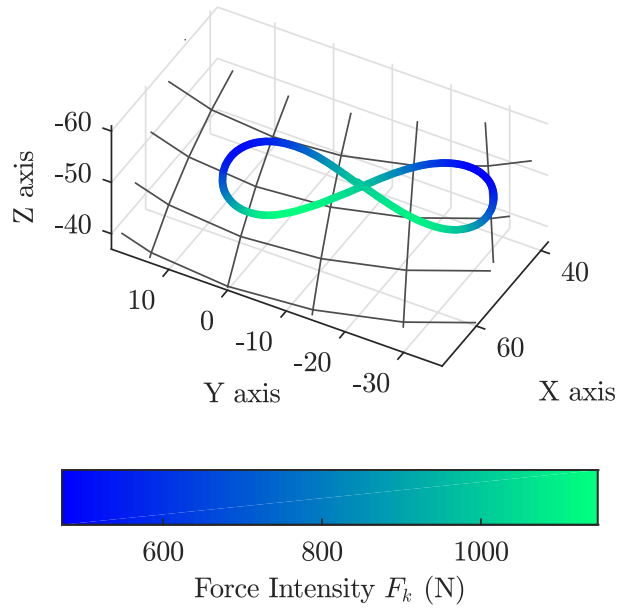

(b)

Figure 10: Evolution of kite load $F_{k}$ along a 8-pattern trajectory, for the reference case (see Tab.1). Plot (a) shows variation of the load over the time, with the associated standard deviations computed thanks to the phase averaging method. Plot (b) shows the same data but according to the position of the kite along the trajectory.

\subsubsection{Extreme loads observed}

During all phases of experiments, higher loading conditions occurred sometimes on the kite at specific moments, as at launch for example. These moments cannot be processed as it has been done it the previous Subsection because, in those cases, the kite is not doing a repetitive regular pattern. However since loads generated during these moments are so much higher than during other phases it definitely has to be investigated. Two cases have been identified, one for each kite.

With the North kite (Fig.12), load exceeded $2500 \mathrm{~N}$ during three peaks for a total duration of $4 \mathrm{~s}$ (for a period duration of 12s), with a maximum force reaching $3700 \mathrm{~N}$, with an average 
true wind speed during the period of $9.02 \mathrm{~m} / \mathrm{s}$. This maximum value is equivalent to a wing load equal $0.74 \mathrm{kN} / \mathrm{m}^{2}$.

With the second kite (Cabrinha), only one peak was observed reaching $3400 \mathrm{~N}$, however the average load during the $7 \mathrm{~s}$ period was slightly above $2000 \mathrm{~N}$, with an average true wind speed during the period of $10.92 \mathrm{~m} / \mathrm{s}$. The evolution of the load during this period is given in Fig.11.

\subsubsection{Buckling Phenomena}

None of the cases presented in Tab. 1 shows a true wind speed higher than $8 \mathrm{~m} / \mathrm{s}$. Nevertheless, such wind conditions occurred during the month of measurement, as it can be seen with the observation of extreme loading cases. However, no run suitable for the phase averaging method could be achieved with these wind conditions. This is due to buckling phenomena occurring on the inflatable leading edge of the kite and also on battens. When the amplitude of the phenomena was limited, it has no important effects, but when the wind speed increased, the amplitude also increased, generating losses of performance and control problems of the kite. Thus, the autopilot was no longer able to draw suitable 8-pattern trajectories, leading to kite tracks appearing like a succession of unpredictable loops with no identifiable pattern. In extreme case, the autopilot was even not able to maintain kite in flight, leading to the crash of the kite, often violently. Examples of instantaneous observations of buckling phenomena are given in Fig.12. Picture (a) presents a very classic case with a little bending at the end of the leading edge. Pictures (b), (c) and (d) show the same phenomena, propagating to the last and middle battens, and affecting also the leading edge at wider scale. Finally, picture (e) presents a case where the kite was totally collapsed, during approximately $150 \mathrm{~ms}$.

Another example of buckling phenomenon can be seen on force records presented in Fig.11. Indeed the force oscillations observed between $0.8 \mathrm{~s}$ and $1.6 \mathrm{~s}$ and between $2.5 \mathrm{~s}$ and 3.3 $s$ are typical of a buckling issue: when load reaches a certain threshold, buckling appears leading to a significant loss of performance of the kite. Then, when kite load is sufficiently low, the kite retrieves a better shape, and load can raise up again, until a new partial collapse. These oscillations continue until the kite reach a reduced power area, like the wind window edge. According to the data, in this case, oscillations occur at approximately $3 \mathrm{~Hz}$. During this type of events, autopilot or manual pilot have almost no control on the kite motion. The other force oscillations observed just before the 4th second and around the 6th second are also probably buckling phenomena, but as no video data are available for this case, it is then difficult to conclude.

Parameters ruling the threshold levels of buckling appearance are complex and not predictable from what we know. Indeed, still in Fig.11, buckling affects load for the first time around $2600 \mathrm{~N}$, whereas one second later the load reaches $3400 \mathrm{~N}$ before being affected. However, two elements have a clear influence on buckling threshold: the pressure of the leading edge and the bridle structure. Pressure effect was clearly observed during fieldwork: when the kite lost pressure due to little holes in the inflatable part, it was more exposed to buckling after a few minutes of flight than just after the take-off. Bridle structure is more complicated to modify and no test was carried out in that way. But it is obvious that these types of kite are not designed to be used onshore by being permanently attached to the ground. Indeed, under normal conditions, a $75 \mathrm{~kg}$ kite-surfer cannot hold out against a force of 1500 $\mathrm{N}$. Then, he will move towards the kite generating a velocity of the attachment point. This velocity will cause a decrease of the angle of attack and of the relative wind velocity on the kite, resulting in a significant reduction of the load. 


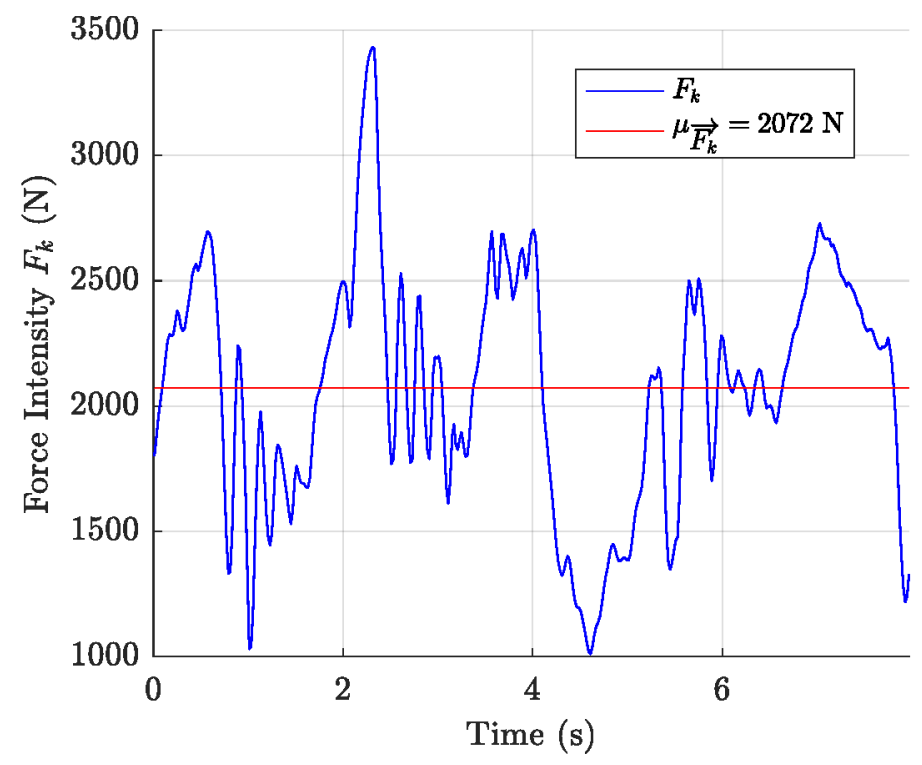

Figure 11: Maximum effort recorded for Cabrinha kite during fieldwork.

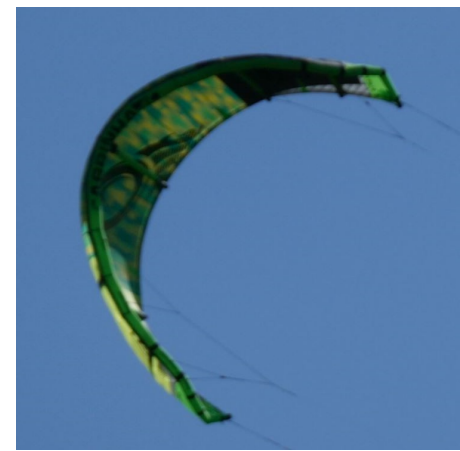

(a)

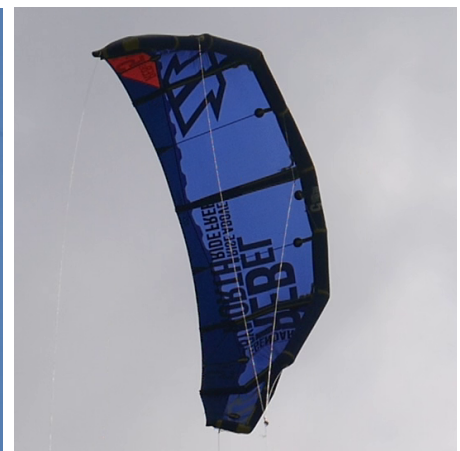

(b)

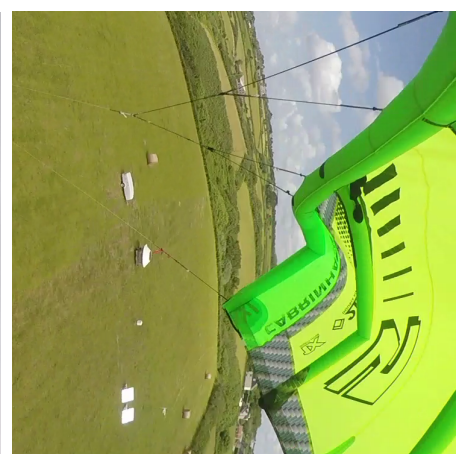

(c)

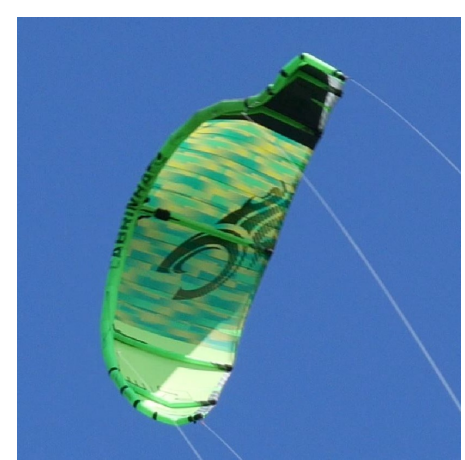

(d)

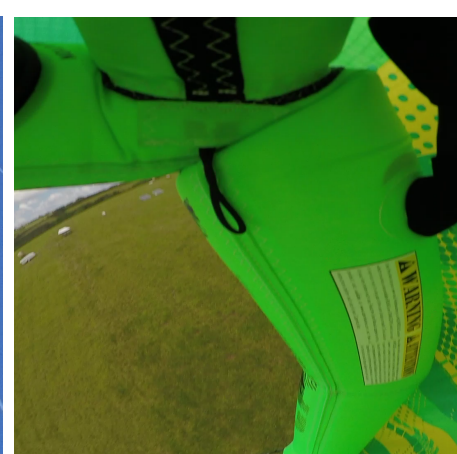

(e)

Figure 12: Pictures of buckling phenomena, with several levels of deformation . Picture (e) shows the junction between the inflatable leading edge and the central batten (the camera was mounted on the central batten, shooting forward). 


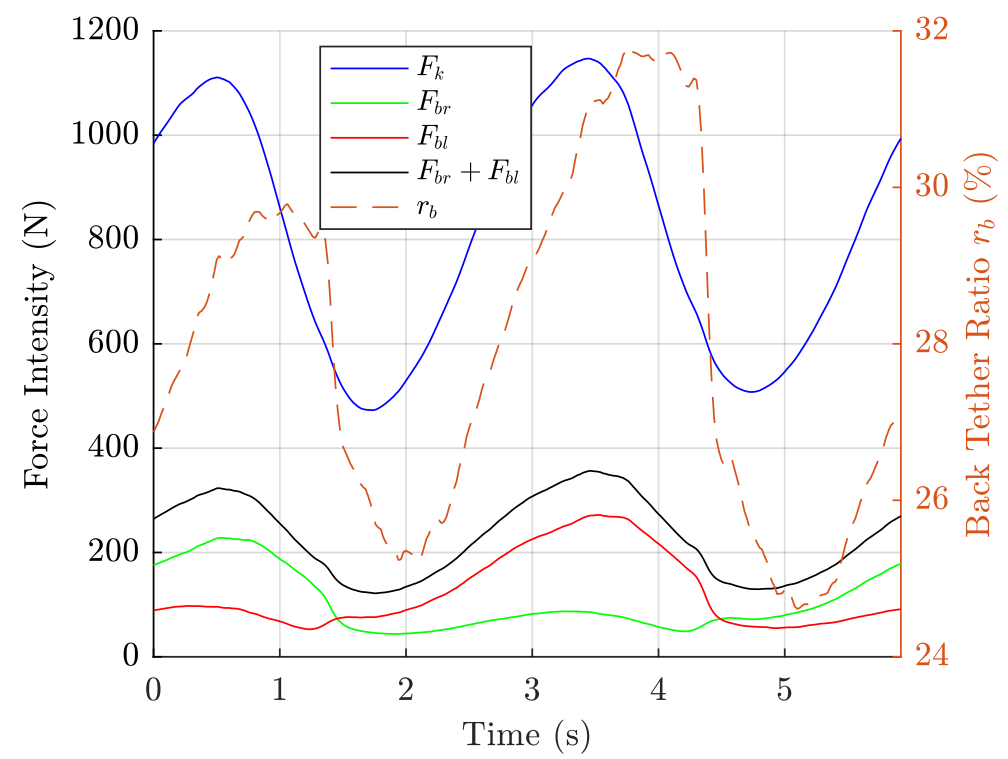

Figure 13: Back tether load versus total load during the reference case.

\subsection{Ratio between forces into back tethers and total force}

For the purpose of commercial development of kite control systems, the part of effort supported by the back tethers is an interesting element to analyze. Indeed this information is essential to size correctly the power of the actuators and to define back tether section and material. As it can be seen in Fig.13, the ratio between the sum of contributions of the back tethers and an the total kite force, as defined in Eq.22, varies between $25 \%$ and $30 \%$ along the trajectory of the reference case. The average values for the complete eight-patterns presented in Tab. 1 are between $18 \%$ and $30 \%$.

\subsection{Impact of trim}

The autopilot was programmed in order to get the steering input $\delta$ independent from the trim $\tau$ (see Subsection 3.2.1). This means that within the same run the trim is kept constant.

The trim $\tau$ of the kite was defined as the length difference between back and front tethers. Thus, if front tethers were directly connected to the attachment point, the trim value should be the distance between real start of back tethers and attachment point. Indeed back tether are all the time longer than front ones. And this distance is measured thanks to the optical encoders. However, between real start of front tether and attachment point, a front leader line was rigged (as shown in Fig. 4 b ), and the length of this leader line was not measured precisely. Moreover, this leader line was replaced several times due to important friction at attachment point. Thus trim values cannot be compared from one day to another. This entails that kites were not rigorously set in the same way all the time, and therefore absolute performances cannot be really compared.

However a record was specifically done to analyze the effect of the trim on kite performances. Thus during a run, with no change of any other parameters, three trim values were tested during a few minutes. Average results are given in Tab.2, and these trim values are expressed relatively to the first one. This measurement was done with the North kite rigged with $52 \mathrm{~m}$ tethers.

Differences between the case 2 and 3 are significant, under similar conditions (only $1.5 \%$ in difference on wind) we measured more than $4.5 \%$ in mean load and $8 \%$ in load coefficient 
Table 2: Impact of trim on kite performances

\begin{tabular}{|c|c|c|c|c|c|c|}
\hline $\begin{array}{c}\text { Case } \\
\text { Name }\end{array}$ & $\begin{array}{c}t_{r} \\
(\mathrm{~s})\end{array}$ & $\begin{array}{c}V_{W T} \\
(\mathrm{~m})\end{array}$ & $\begin{array}{c}\mu_{F_{k, A}} \\
(\mathrm{~N})\end{array}$ & $\begin{array}{c}C_{k} \\
(-)\end{array}$ & $\begin{array}{c}r_{b} \\
(\%)\end{array}$ & $\begin{array}{c}\triangle \tau \\
(\mathrm{cm})\end{array}$ \\
\hline \hline $\mathrm{BC} 1$ & 350 & 6.2 & 959.4 & 7.8 & 21 & 0 \\
\hline $\mathrm{BC} 2$ & 58 & 6.4 & 1729.7 & 13.2 & 25 & -4 \\
\hline $\mathrm{BC} 3$ & 290 & 6.5 & 1654.9 & 12.2 & 25 & -5 \\
\hline
\end{tabular}

$\mathrm{Ck}$, but trim difference is only $1 \mathrm{~cm}$, and case 2 deals with a time window $t_{r} 5$ times shorter. However the comparison between case 1 and case 3 is more appropriate (similar period length). Therefore, a trim reduction of $5 \mathrm{~cm}$ leads to an increase of kite force about $72 \%$. On the same time the back tether force ratio goes from $21 \%$ to $25 \%$.

\subsection{Apparent wind versus true wind speed}

When the kite is flying in dynamic mode with $\left\|\underline{V}_{k}\right\|>0$ (i.e. not in static mode), most of the time, the kite velocity increases the apparent wind seen by the kite. And this is one of the major advantages of kites in comparison with classic sails, which are fixed to the boat. Thus, it is interesting to analyze the factor between the relative wind speed, equals in the current fieldwork to the true wind speed, with the apparent wind. Therefore, this average factor was computed for each of the 25 cases presented in Tab.1, and then averaged again for all cases. Finally, the average factor is 3.1. Because forces are related to the square of the apparent wind speed, this value shows that kites used in our study are almost 10 times more powerful than a classic sail with the same size. But this is only true when the kite is operated in dynamic flight. In static flight, the apparent wind is equal to the true wind, and kites in this case are equivalent to sails.

\subsection{Kite model impact on post processing}

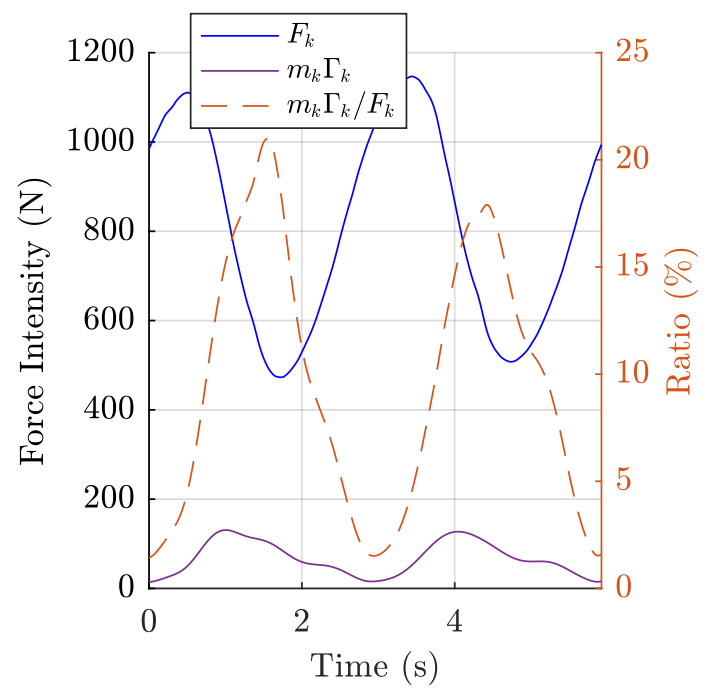

(a)

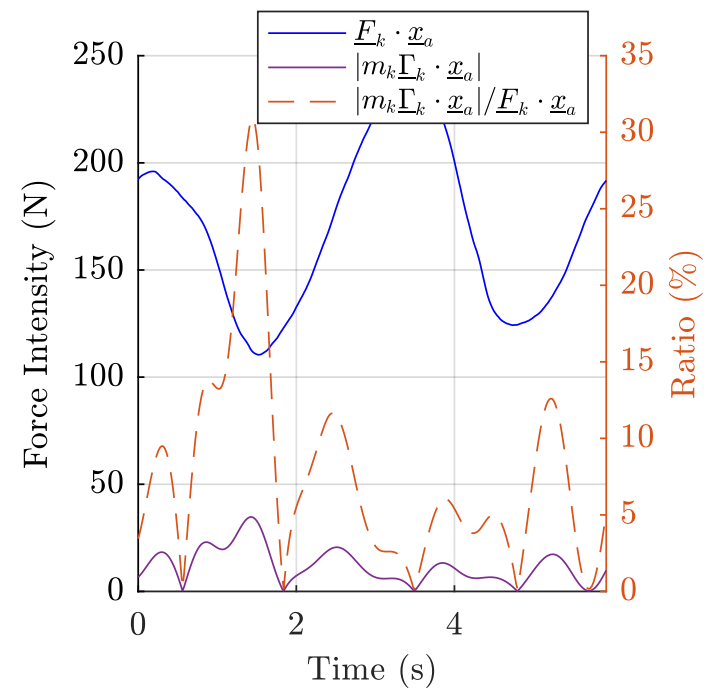

(b)

Figure 14: Comparison between the total kite force and inertial forces including weight, during the reference case (Tab.1). Plot (a) deals with the norm of these forces, whereas plot (b) is a focus on the projection of these forces on the apparent wind axis. 
Two different models have been presented in Eq.7 and Eq.8 to estimate the aerodynamic force of the kite. The first one is the zero-mass model assuming the kite has no mass and then no inertial effect exists. The second model is the point-mass model, assuming the kite mass is concentrated at point $K$. This entails the apparition of inertial forces and weight. In Fig.14a, the total kite force is plotted with the calculated inertial effect. We set the mass of the kite to $3 \mathrm{~kg}$ for this calculation. The ratio between these two values is printed on the right axis. Even if the inertial effect remains low, they can reach nonetheless $20 \%$ of the kite force value. When projected on the wind apparent axis $\underline{x}_{a}$ as shown in part (b) of Fig.14, the inertial effect amounts as much as $30 \%$ of the total kite force projected on $x_{a}$-axis, in other words, the drag of the zero-mass model. Therefore, the choice of the model will particularly affect the drag calculation, and so the lift to drag ratio result. This will be confirmed by the computation of the lift to drag ratio and the lift coefficient with one of the two models in the two following Subsections.

\subsection{Lift to drag ratio}

The lift to drag ratio has been computed using Eq.14, and the time evolution of lift to drag value along the trajectory of the kite during the reference case is given in Fig.15a. Three lines are plotted. The blue one is the lift to drag ratio calculated from wind data coming from the SODAR and using the zero-mass model. The orange one uses also SODAR data, but with the point mass model. Finally the yellow one is based on METEK wind data with the zero-mass model. An important difference is observed between the last line (METEK data yellow line) and the two others (SODAR data - blue/orange line). This can be easily explained regarding average wind speed given by the two different devices: thus the wind measured by the SODAR at kite altitude is about $5.0 \mathrm{~m} / \mathrm{s}$ whereas the wind measured at the time by the METEK sensor with no profile compensation was $3.3 \mathrm{~m} / \mathrm{s}$. This shows how important it is to know as well as possible the wind at kite altitude for post-processing lift to drag ratio. The average value of the lift to drag ratio computed with the point mass model is 4.6. Plot (b) of Fig. 15 shows the same lift to drag data, but plotted in relation to the rotation speed of the velocity vector of the kite $\underline{V}_{k}$ around tethers axis. According to what was discussed before, the line with METEK data is not plotted. 


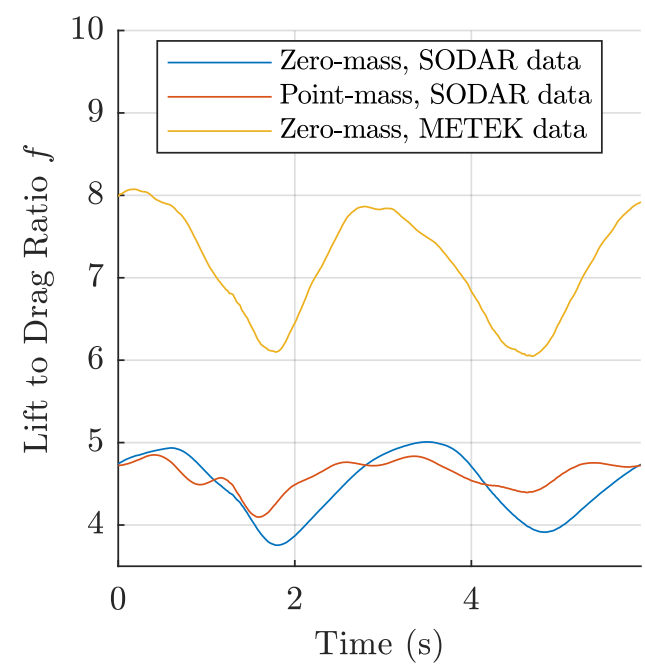

(a)

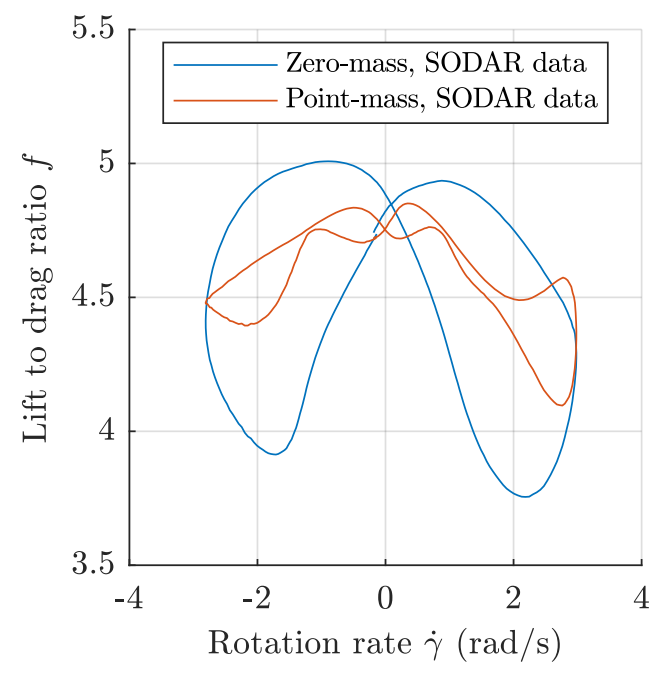

(b)

Figure 15: Evolution of the lift to drag ratio along the trajectory, for the reference case (Tab.1). Plot (a) gives the time evolution, and plot (b) gives the evolution of the ratio according to the rotation speed of the kite velocity vector around the tether axis.

\subsection{Lift coefficient}

The lift coefficient of the kite computed using Eq.15 has been processed in the same way as for the lift to drag ratio. Thus, time evolution of lift coefficient is given in Fig.16a, and the evolution related to $\dot{\gamma}$ is given in plot (b). It is interesting to notice that true wind speed value has less influence for lift coefficient computation than for the lift to drag ratio. Indeed, difference between results with METEK data and SODAR data is lower. Indeed, the true wind has much effect on the lift to drag ratio because it tilts the aerodynamic reference frame. As for the lift to drag ratio, the lift coefficient is getting lower at high turn rate, positive or negative. The average value of the lift coefficient for the whole period is 0.8 . 


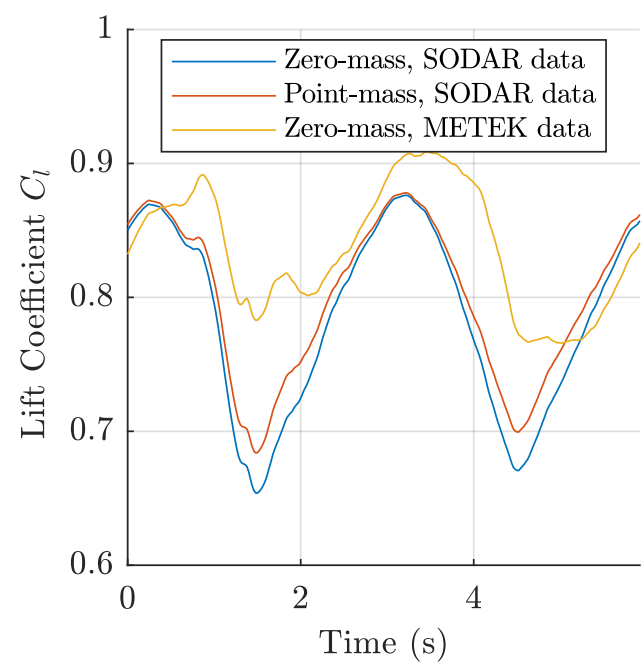

(a)

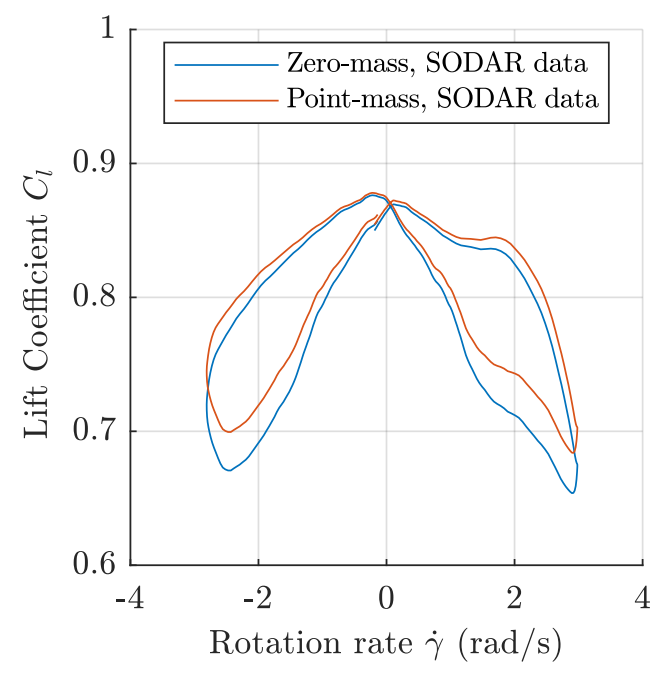

(b)

Figure 16: Evolution of the lift coefficient along the trajectory, for the reference case (Tab.1). Plot (a) gives the time evolution, and plot (b) gives the evolution of the coefficient according to the rotation speed $\dot{\gamma}$ of the kite velocity vector around the tether axis.

\subsection{Wind variability impact}

The SODAR used give a measurement of the averaged wind as a function of altitude on a five-minute interval basis. The true wind variability is therefore not included in the phase averaging process (the kite trajectory had a pseudo-period of approximately $6 \mathrm{~s}$ in the reference case shown in Tab. 1). However, the SODAR measures also the standard deviation of the different components of the true wind. Based on this it is possible to compute a posteriori the impact the true wind variability would have on the presented results. For this purpose, a simple Monte Carlo method was used to reproduce the averaged phase method by adding a Gaussian noise on the three components of the measured true wind. The variations of these three components are supposed to be independent of each other. The Monte Carlo scheme is repeated until a satisfactory statistic is reached for the computation of the average value and the standard deviation. Results obtained are presented in Fig.17. The impact of true wind variability on the lift coefficient is weak and can therefore be neglected once the repeatability is good enough as for the reference case for instance. The impact of true wind variability on the lift to drag ratio is more important but remains moderate (slight increase of the average value of about $0.7 \%$ with a standard deviation doubled by reaching approximately $1 \%)$.

\section{DISCUSSION}

The lift to drag ratio and the lift coefficient plotted in Fig.15 and 16 are the result of the phase averaging method, leading to data coming from 75 8-patterns (regarding the reference case of Tab.1) and averaged to get only one pattern. Therefore the observed variations have a physical signification. The standard deviation from the phase averaging method gives an illustration of the uncertainty in Fig.10 as an example. Nevertheless, better results could be expected in the future, with a new set of data. A systematic variation of kite specifications could be highlighted, with respect to the rotation rate of the velocity vector of the kite around tether axis, or with respect to other quantities. 


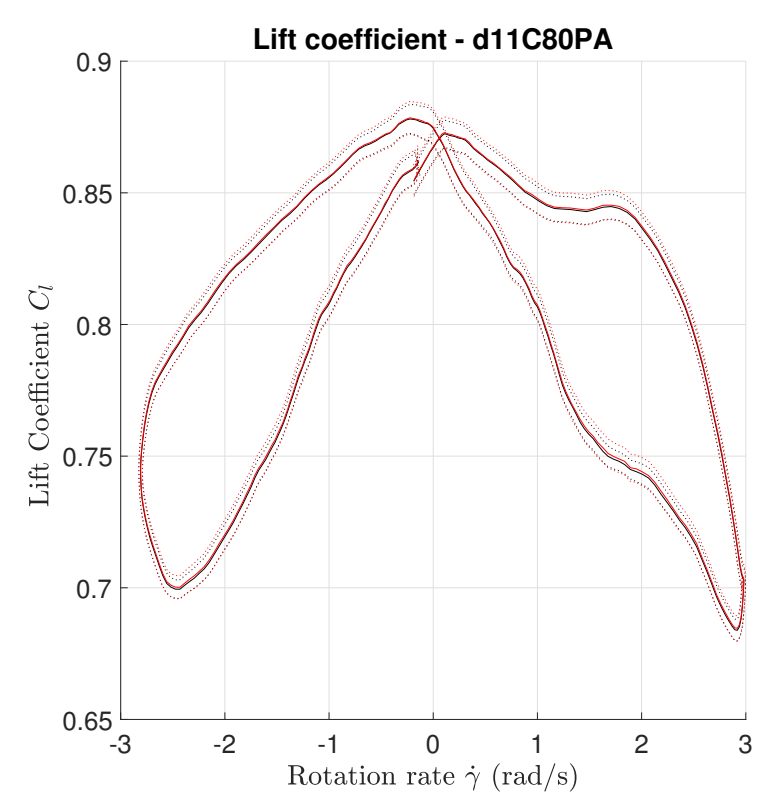

(a)

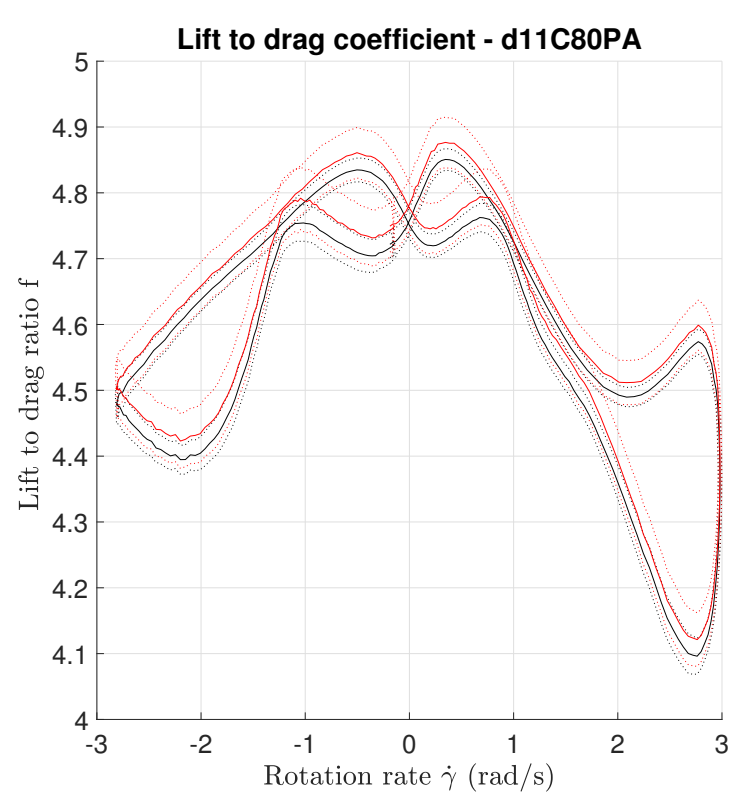

(b)

Figure 17: Effects of a Gaussian noise, with (red) and without (black), for the wind variability a posteriori simulation, standard deviation same as the one measured by the SODAR. Light dotted lines represent $\pm 2 \sigma_{\mu}$.

Consequently, these future works should have to focus on several points. At first the symmetry issues have to be considered carefully, with a rigorous verification of all elements that can lead to potential symmetry issue. For example tether lengths should be carefully checked, and particularly the lengths of left and right tethers. These measurements must be carried out at various loads. For that, the two tethers are fixed on one side, and the other sides of the two tethers are linked using a leader line. This leader line goes through a return pulley, and a load is applied to the pulley. Therefore, the load is equally split between the two tethers. When the applied load rises, the length difference should not change if the two tethers have experienced the same history. However, when operating the kite, during a hard turn or during a crash, a tether could be slightly damaged, and then the elongation under load can be different from one to another tether. To identify symmetry issues the data post processing should be done quickly.

Secondly, new sensors could be added to bring more information about the kite position and kite attitudes. For example, IMUs could be embedded on the kite again, but only for estimating the yaw angle of the kite, and to compare this value to the angle $\gamma$ related to the velocity vector. The yaw angle of the kite could also be obtained using video tools, by filming the kite from the ground, and performing an image analysis. This solution could also lead to a measurement of the flying shape, if several camera are used, associated with a photogrammetry post processing. This possibility, already tested by Infanzon (2013) with limited results, can probably be improved with better cameras. Much more could also be done regarding embedded cameras, to get a finest understanding of phenomenon occurring during turns. For example, yarn tell-tales could be stuck on the kite, and filmed by a camera. Thus, separated flows could be observed at some locations, as it can be done for the analysis of a stall occurring on the wing of an airplane ${ }^{1}$.

Thirdly, a simple aerodynamic modeling of the kite could be achieved, based for example on a

\footnotetext{
${ }^{1}$ https://www.youtube.com/watch?v=WFcW5-1NP60
} 
non-linear lifting line method (Leloup et al., 2013), (Duport et al., 2015), to foresee kite specs during turns, and then provide a guidance on the expected variations of the aerodynamic parameters of the kite.

The lift to drag ratio and the lift coefficient variations along an 8-pattern trajectory are not brought out in a perfect conclusive way by the present measurements. However, these variations exist, and lead consequently to larger amplitudes of forces than the ones predicted by the zero-mass model. These amplitudes have to be considered: indeed, they can be detrimental to boat stability, actuator power or material wear if they are not taken account. Therefore it has been decided to propose an evolution law based on the average of the reference case (blue row in Tab.1) and on two other similar cases (C80_1 and C80_2 in Tab.1). In this way, modeling of kite and modeling of boats towed by kite could integrate these variations, and these laws will be updated when further works lead to results with better accuracy. To get rid of the symmetry issue, data over the 8-pattern trajectory have been processed in order to make them symmetrical. For this purpose, each signal (lift to drag ratio $f$ and lift coefficient $C_{l}$ ) has been cut into two pieces of equal length, and these two pieces have then been averaged. This is only possible because the middle of the 8-pattern corresponds to the middle of the time-series. Thus, for a signal $S$ defined for $t \in \llbracket 0, t_{a} \rrbracket$, the symmetrical signal $S_{\text {sym }}$ is:

$$
S_{\text {sym }}(t)= \begin{cases}\frac{S(t)+S\left(t+\frac{t_{a}}{2}\right)}{2} & t \in \llbracket 0, \frac{t_{a}}{2} \rrbracket \\ \frac{S\left(t-\frac{t_{a}}{2}\right)+S(t)}{2} & t \in \rrbracket \frac{t_{a}}{2}, t_{a} \rrbracket\end{cases}
$$

Results of this process are given in Fig.18. Only positive rotation rates are plotted, because the signal is now symmetrical about the axis defined by $\dot{\gamma}=0 \mathrm{rad} / \mathrm{s}$. This leads to the following linear laws:

$$
\begin{gathered}
f=4.83-0.16|\dot{\gamma}| \\
C_{l}=0.87-0.05|\dot{\gamma}|
\end{gathered}
$$

The symmetrical data set used for proposing these laws is also used by Bigi et al. (2018). In this work, linear laws are also proposed, but the parameters are obtained in a different way: the experimental data are compared with zero-mass model results, and the parameters are adjusted to get the best fit possible. The parameter values obtained are close but no equal. Indeed the current laws Eq.27 and Eq.28 for the lift to drag ratio and the lift coefficient are computed from a point-mass model, as it is explained Section 5.5; and thus they should be used within the same assumption.

\section{CONCLUSION}

A kite experimental setup has been developed to be used onshore or embedded onboard of a dedicated boat. A 3D load cell is used to get kite force and its position into the wind window. The kite is controlled using winches, and an autopilot performs repeatable figure-ofeight trajectories. During the onshore campaign held in June 2016, a sonic wind profiler was used to estimate the wind at kite altitude. Buckling phenomena were observed. The link was established with flight instabilities and control issues. A point mass model and a zero mass model were investigated for the post processing of the data. The influence of kite inertia and weight on the computed drag and lift to drag ratio was highlighted, resulting to a drop about $20 \%$ during the turns in the presented case. Then, insufficiencies of the zero mass model were pointed out. The phase averaging method was used to post process a selected part of 


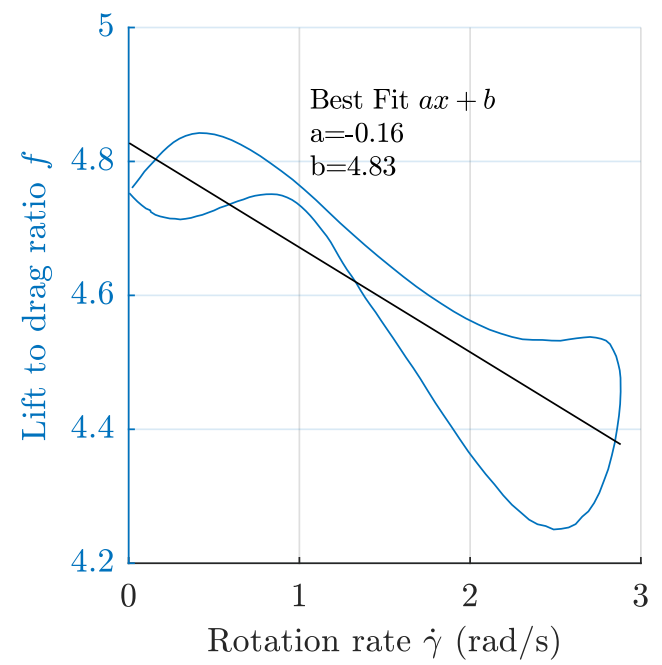

(a)

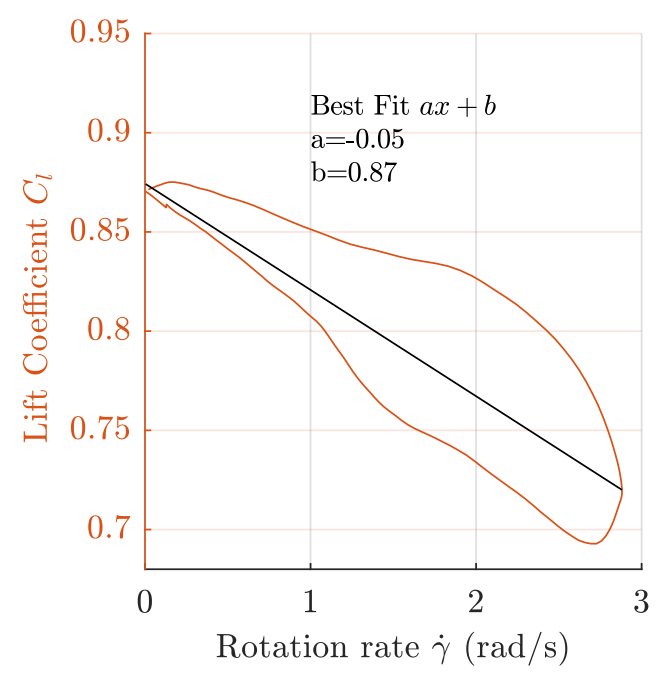

(b)

Figure 18: Best fit processed on symmetrical signal of the lift to drag ratio (a) and the lift coefficient (b), from an average data set coming from three cases (C80_1, C80_2 and C80_3 in Tab.1)

data obtained during this campaign in order to get more accurate and reliable results. This method allows estimating evolution laws regarding the lift coefficient and lift to drag ratios as functions of the turning rate of the kite.

Future work will focus on onboard data that will be also post processed, using the phase averaging presented here.

\section{ACKNOWLEDGEMENTS}

The authors are grateful to the French Environment \& Energy Management Agency (ADEME) for funding this study.

\section{References}

Behrel, M., N. Bigi, K. Roncin, D. Grelon, F. Montel, A. Nême, J.-B. Leroux, C. Jochum, and Y. Parlier (2016). Measured Performance of a 50-m2 Kite on a Trawler. In 10th Symposium on High-Performance Marine Vehicles (Hiper), Cortona, ITALY, pp. 443-457.

Behrel, M., K. Roncin, J. B. Leroux, F. Montel, A. Nême, C. Jochum, and Y. Parlier (2017). Experimental set up for measuring onshore and onboard performances of leading edge inflatbale kites - presentation of onshore results. In Fourth International Conference on Innovation in High Performance Sailing Yachts (INNOV'SAIL), Lorient, FRANCE.

Bigi, N., M. Behrel, K. Roncin, J.-B. Leroux, A. Neme, C. Jochum, and Y. Parlier (2018). Dynamic modeling of ships towed by kite. Houille Blanche 2018-Febru(1), 8-10.

Bormann, A., M. Ranneberg, P. Kövesdi, C. Gebhard, and S. Skutnik (2013). Development of a Three-Line Ground Actuated Airborne Wind Energy Converter. In U. Ahrens, M. Diehl, and R. Schmehl (Eds.), Airborne Wind Energy, Chapter 24, pp. 427-441. Berlin: Springer Berlin Heidelberg. 
Dadd, G. M. (2013). Kite dynamics for ship propulsion. Ph. D. thesis, University of Southampton.

de Solminihac, A., A. Nême, C. Duport, Jean-Baptiste, K. Roncin, C. Jochum, and Y. Parlier (2018). Kite as a beam: A fast method to get the flying shape. In R. Schmehl (Ed.), Airborne Wind Energy - Advances in Technology Development and Research, Chapter 24, pp. 427-441. Singapore: Springer.

Duport, C., J.-B. Leroux, K. Roncin, C. Jochum, and Y. Parlier (2015). Comparison of 3D non-linear lifting line method calculations with 3D RANSE simulations and application to the prediction of the global loading on a cornering kite. In 15th Journées de l'Hydrodynamique, Brest, FRANCE.

Erhard, M. and H. Strauch (2013). Control of towing kites for seagoing vessels. IEEE Transactions on Control Systems Technology 21(5), 1629-1640.

Fagiano, L., K. Huynh, B. Bamieh, and M. Khammash (2013). Sensor fusion for tethered wings in airborne wind energy. 2013 American Control Conference (ACC), 2884-2889.

Fagiano, L., K. Huynh, B. Bamieh, and M. Khammash (2014). On sensor fusion for airborne wind energy systems. IEEE Transactions on Control Systems Technology 22(3), 930-943.

Fagiano, L. and T. Marks (2015). Design of a small-scale prototype for research in airborne wind energy. IEEE/ASME Transactions on Mechatronics 20(1), 166-177.

Infanzon, R. (2013). Photogrammetry Based Investigation of a Kite's Flying Shape - PPT. Technical report, Australian Maritime College, Launceston.

ISO 1151-1-4 (1988). Flight dynamics - Concepts, quantities and symbols - Part 1: Aircraft motion relative to the air. Technical report, ISO, Geneve, SWITZERLAND.

ITTC Quality Group, S. (2014). ITTC Symbols and Terminology List. In International Towing Tank Conference, Copenhagen, DENMARK.

Leloup, R., K. Roncin, and M. Behrel (2016). A continuous and analytical modelling for kites as auxiliary propulsion devoted to merchant ships, including fuel saving estimation. Renewable Energy 86, 483-496.

Leloup, R., K. Roncin, G. Bles, J.-B. Leroux, C. Jochum, and Y. Parlier (2013). Estimation of the lift-to-drag ratio using the lifting line method: Application to a leading edge inflatable kite. In U. Ahrens, M. Diehl, and R. Schmehl (Eds.), Airborne Wind Energy, Chapter 19, pp. 339-365. Berlin: Springer-Verlag Berlin Heidelberg.

Naaijen, P., V. Koster, and R. P. Dallinga (2006). On the power savings by an auxiliary kite propulsion system. International Shipbuilding Progress 53(4), 255-279.

Sagaut, P. (2006). Large eddy simulation for incompressible flows: an introduction. Springer Science \& Business Media.

Wellicome, J. F. (1985). Some comments on the relative merits of various wind propulsion devices. Journal of Wind Engineering and Industrial Aerodynamics 20(1-3), 111-142.

Wernert, P. and D. Favier (1999). Considerations about the phase averaging method with application to ELDV and PIV measurements over pitching airfoils. Experiments in Fluids 27(6), 473-483. 
Yu, J., L. liu Shi, W. zhe Wang, and Y. zheng Liu (2010). Conditional averaging of TRPIV measurements of wake behind square cylinder using an improved cross-correlation approach. Journal of Hydrodynamics 22(1), 29-34. 